PALEO

Revue d'archéologie préhistorique

$20 \mid 2008$

Spécial table ronde ( $2^{\mathrm{e}}$ partie) : Le Gravettien : entités régionales d'une paléoculture européenne, Les Eyzies, juillet 2004

\title{
Le Gravettien et le Protomagdalénien en Auvergne
}

Gravettian and Protomagdalenian in Auvergne region

Frédéric Surmely, Sandrine Costamagno, Maureen Hays, Philippe Alix et Jean-François Pasty

\section{OpenEdition}

Journals

Édition électronique

URL : http://journals.openedition.org/paleo/1598

DOI : $10.4000 /$ paleo. 1598

ISSN : 2101-0420

Éditeur

SAMRA

Édition imprimée

Date de publication : 1 décembre 2008

Pagination : 305-330

ISSN : $1145-3370$

Référence électronique

Frédéric Surmely, Sandrine Costamagno, Maureen Hays, Philippe Alix et Jean-François Pasty, « Le Gravettien et le Protomagdalénien en Auvergne », PALEO [En ligne], 20 | 2008, mis en ligne le 12 mai 2011, consulté le 07 juillet 2020. URL : http://journals.openedition.org/paleo/1598 ; DOI : https:// doi.org/10.4000/paleo.1598

\section{(c) (i) () $९$}

PALEO est mis à disposition selon les termes de la licence Creative Commons Attribution - Pas d'Utilisation Commerciale - Pas de Modification 4.0 International. 


\title{
LE GRAVETTIEN ET LE PROTOMAGDALÉNIEN EN AUVERGNE
}

\author{
Frédéric SURMELY ${ }^{(1)}$, Sandrine COSTAMAGNO( ${ }^{(2)}$, \\ Maureen HAYS ${ }^{(3)}$ et Philippe ALIX ${ }^{(4)}$
}

Avec la collaboration de Jean-François PASTY ${ }^{(5)}$

\begin{abstract}
Résumé : Le Gravettien et le Protomagdalénien ne sont connus en Auvergne que sur deux ou trois sites, ce qui est fort peu à l'échelle d'une région qui couvre plus de $26000 \mathrm{~km}^{2}$. Cela semble imputable à des raisons taphonomiques, dans une région marquée par un climat rude ainsi que par un faible développement des recherches. Le gisement du Sire (Mirefleurs, Puy-de-Dôme) est un vaste site de plein air qui a livré deux riches niveaux d'occupation datés du Gravettien ancien (circa 30000 BP). Le Gravettien récent et le Protomagdalénien ont été reconnus sur le site du Blot (Cerzat, Haute-Loire) qui présente une stratigraphie complexe, avec de nombreux niveaux archéologiques correspondant probablement à des occupations répétées et de courte durée. Le Protomagdalénien du Blot offre des caractères spécifiques (comme la recherche d'un débitage de grandes lames) qui le distinguent nettement du Gravettien. Les gisements d'Auvergne se caractérisent par un recours systématique à des matières premières d'origine lointaine, parmi lesquelles figurent le silex du Grand-Pressigny et le silex de la craie du Turonien inférieur. Ils sont marqués également par l'abondance des armatures microlithiques (microgravettes et lamelles à dos) et l'absence de projectiles en matière animale.
\end{abstract}

Mots-clés : Gravettien, Périgordien, Protomagdalénien, Auvergne, Paléolithique supérieur, matières premières lithiques, microlithes.

Abstract: Gravettian and Protomagdalenian in Auvergne region. The Gravettian and Protomagdalenian are known in the Auvergne region from only two or three sites, which is rather sparse for a region extending over more than 26,000 square kilometers. Some of this may be accounted for by taphonomy in an under researched area characterized by a harsh climate. The vast open-air site of Le Sire (Mirefleurs, Puy-de-Dôme) has two rich occupational levels dated to the early Gravettian (C. 30,000 BP). The late Gravettian and Protomagdalenian are known from the site of Le Blot (Cerzat, Haute-Loire). Le Blot has a complex stratigraphy with many archaeological levels possibly corresponding to repeated occupations of short duration. The Protomagdalenian from Le Blot has distinct features (for example extremely long blades) which clearly distinguish it from the Gravettian. The main characteristics for all Auvergne sites is the systematic exploitation of raw material from distant sources (for example Grand-Pressigny flint and early Turonian chalk flint), an abundance of small projectile points (microgravettes and blacked bladelets), and the absence of projectiles produced from faunal material.

Key-words: Gravettian, Perigordian, Protomagdalenian, Auvergne, Upper Palaeolithic, raw material, microliths.

\section{INTRODUCTION}

Le Gravettien n'est connu en Auvergne que sur deux ou trois sites, ce qui est fort peu à l'échelle d'une région qui couvre plus de $26000 \mathrm{~km}^{2}$, soit $5 \%$ du territoire français.
L'Auvergne est une région dont les traits caractéristiques sont sa position centrale, entre Bassin parisien, Midi, Aquitaine et Bassin rhodanien, la présence de reliefs accusés, avec un bassin central (La Limagne) encadré par des moyennes montagnes culminant à 1800 m et un climat assez rude. Le milieu environ-

(1) Geolab, UMR 6042, Maison de la Recherche, université de Clermont-Ferrand, 4, rue Ledru 63000 Clermont-Ferrand surmely.frederic@wanadoo.fr

(2) UTAH, UMR 5608, Maison de la Recherche, Université Toulouse Le Mirail, 5 allées A. Machado, 31058 Toulouse Cedex 9 costamag@univ-tlse2.fr

(3) College of Charleston, Department of Anthropology, 19 Saint Philip Street, Charleston, SC 29424, USA - haysm@cofc.edu

(4) INRAP Rhône-Alpes/Auvergne - 12 rue Maggiorini 69500 BRON

(5) INRAP Auvergne - 13b rue Pierre Boulanger - Le Brézet 63100 Clermont-Ferrand 
nant est peu favorable à la bonne conservation des sites préhistoriques. L'érosion, particulièrement active dans une région toute entière marquée par la rudesse du climat, l'ampleur des précipitations et l'importance des pentes, a eu un effet destructeur sur les habitats. Les cavités véritables sont très rares. Les témoins d'origine organique (ossements, pollens) sont rarement conservés. La présence des glaciers, l'activité volcanique tardiglaciaire et holocène dans certains secteurs ont contribué au démantèlement ou à l'enfouissement profond des sites würmiens. Ajoutons enfin que la recherche préhistorique a été moins active que dans d'autres régions.

\section{LE TRÈS PETIT NOMBRE DE SITES}

Les sites gravettiens et protomagdalénien connus actuellement en Auvergne sont, nous l'avons vu, au nombre de deux ou trois. Aucun gisement n'est actuellement recensé dans les départements de l'Allier et du Cantal, malgré quelques indices incertains (découverte de pièces lithiques isolées évoquant une présence gravettienne). Dans le département du Puy-de-Dôme, le seul site connu à ce jour est celui du Sire, sur la commune de Mirefleurs (Puy-deDôme), avec une séquence attribuable à une phase ancienne de cette civilisation. Enfin, le département de la Haute-Loire abrite deux gisements, dont l'un, Le Blot (Cerzat, Haute-Loire), a livré des niveaux attribuables au Gravettien moyen ou récent et au Protomagdalénien.
Il convient de souligner que cette faible présence gravettienne n'est pas propre à la période considérée, mais s'étend à toute la partie ancienne et médiane du Paléolithique supérieur. L'Aurignacien est presque totalement absent, avec seulement quelques pièces figurant dans les séries des fouilles anciennes du site de Châtelperron (Allier ; Delporte et al. 1999). Quant au Solutréen, il est inconnu dans la région. Beaucoup d'auteurs ont attribué ces carences à la rudesse du climat auvergnat qui aurait créé un environnement naturel peu attirant, voire carrément inaccessible pour l'homme, alors même qu'Aurignacien, Gravettien et Solutréen sont assez bien représentés dans les régions périphériques (Corrèze, sud du Bassin parisien, Ardèche, Bourgogne...), y compris par des grottes ornées. Nous discuterons de cette hypothèse dans la conclusion.

\section{LES DÉBUTS DU GRAVETTIEN : LE SITE DE PLEIN AIR DU SIRE À MIREFLEURS (PUY-DE-DÔME)}

\section{Présentation du site}

Découvert en 1969 par Pierre Daniel, à l'occasion de travaux agricoles, le site avait d'abord été attribué au Magdalénien supérieur. A la suite de la reprise des recherches, à partir de 2000 , sous notre direction, la datation et l'intérêt du gisement ont pu être reconsidérés.

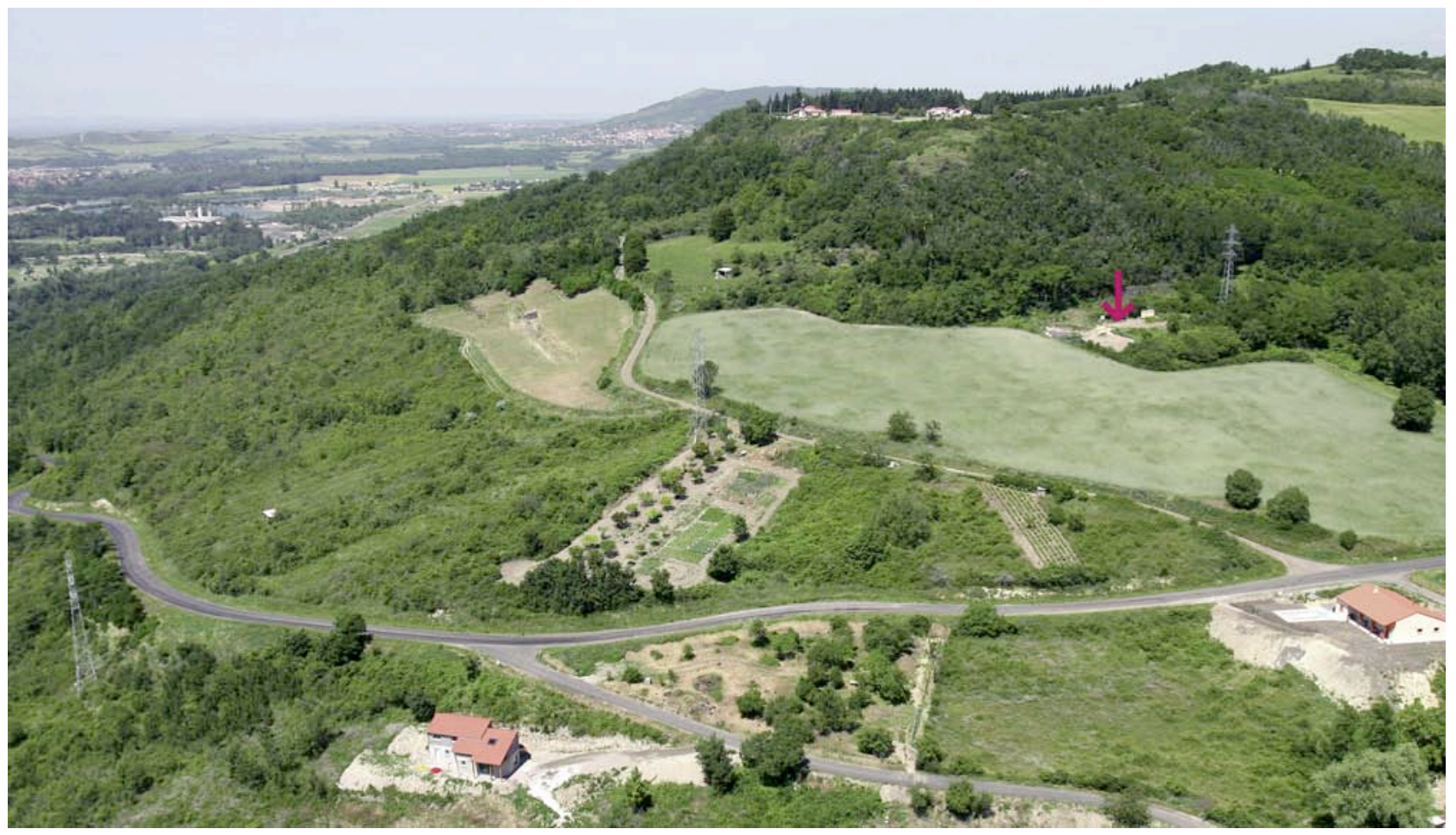

Figure 1 - Le Sire (Mirefleurs, 63) - Gravettien ancien. Vue générale du site gravettien (indiqué par la flèche rouge). Le site moustérien se trouve au premier plan (lotissement). Photo F. Surmely.

Figure 1 - Le Sire (Mirefleurs, 63) - Early gravettian. General view of the gravettian site (indicated by the red arrow). The mousterian site is in the foreground (allotment). Photo F. Surmely. 
Le gisement s'étend sur le versant d'une petite butte calcaire dominant la vallée de l'Allier, au pied des escarpements formés par la coulée de la Roche-Noire (d'âge pliocène), sur un substrat marno-calcaire d'âge oligocène supérieur (fig. 1). La situation géographique semble particulièrement propice à une installation : exposition au midi, proximité des escarpements rocheux, large vue sur la vallée de l'Allier qui est un axe de circulation naturel, proximité des affleurements carbonatés d'âge oligocène offrant d'abondantes ressources en silex (Surmely 1998 ; Dufresne 1999) et de sources minérales salées (Surmely 2004). Ce secteur de la moyenne vallée de l'Allier a d'ailleurs été intensément fréquenté au cours du Paléolithique supérieur et de l'Epipaléolithique, comme le montre la forte densité de gisements connus dans un rayon de quelques kilomètres (Surmely et al. 2003). Enfin, en contrebas du site, sur la même commune, se trouve un très important gisement moustérien, les Chausses, dont la datation pourrait être placée aux environs de 37000 BP (Pasty et al. soumis).

De 2000 à 2002, les opérations se sont limitées à des sondages, destinés à caractériser le site. À partir de 2003, les investigations ont été orientées vers un décapage d'une surface d'environ $60 \mathrm{~m}^{2}$.

Les sondages, même s'ils n'ont pas permis de reconnaître les limites précises du site, montrent que son extension dépasse les $5000 \mathrm{~m}^{2}$.

\section{Stratigraphie et organisation spatiale}

Le site a été affecté par des remaniements d'âge moderne, liés à la mise en culture en terrasses du versant qui ont bouleversé les niveaux sommitaux. La plasticité des sédiments marno-calcaires et la pente affectant le gisement ont favorisé la dispersion verticale des artefacts archéologiques. La base des niveaux archéologiques n'a pas été atteinte à ce jour. Les opérations réalisées, à l'issue de la campagne de 2005, montrent la présence de deux niveaux archéologiques, enveloppés dans des colluvions argilo-limoneuses contenant de nombreux blocs issus du démantèlement de la coulée basaltique sommitale. Dans certains secteurs, les deux niveaux sont difficilement différenciables. II faudra attendre la suite des recherches pour préciser les choses et opérer des distinctions plus tranchées. Faute de sondages profonds, l'existence éventuelle de couches archéologiques sousjacentes n'a pu être vérifiée, mais reste très probable.

Une étude micromorphologique préliminaire a été effectuée par J.-P. Texier. Une seconde étude est en cours (C. Ballut).

Les investigations n'ont pas permis de mettre en évidence des structures nettes, en dehors de zones rubéfiées indiquant des foyers altérés par l'érosion. Une étude est en cours sur la répartition spatiale des vestiges. On note l'existence d'amas de vestiges, principalement osseux, évoquant par endroits le célèbre « magma » d'ossements de Solutré.

\section{Datations}

Les datations, au nombre de 15 (dont trois n'ont pas donné de résultat) ont été faites par AMS, sur ossements animaux. Cinq laboratoires sont intervenus : Waïkato (NouvelleZélande), Oxford (Angleterre), Beta Analytic, Tucson (EtatsUnis d'Amérique) et Poznan (Pologne). Les datations, qui s'échelonnent de 27300 à $31305 \mathrm{BP}$ se répartissent en deux groupes : 27000 / 28000 BP pour le niveau supérieur et $30000 / 31000$ BP pour le niveau inférieur. Bien qu'anciennes, elles confirment le diagnostic typo-technologique en rattachant l'ensemble du site au Gravettien ancien.

Si l'on admet le schéma actuel de variabilité climatique du Paléolithique supérieur élaboré par Heinrich, ces datations placent l'occupation du site dans une période située en dehors des phases de péjoration climatique d'Heinrich 4 et Heinrich 3 (Heinrich 1988 ; d'Errico et al. 2001). Cette hypothèse reste toutefois à confirmer par les avancées récentes de la paléoclimatologie du dernier épisode glaciaire et les problèmes induits par la faible résolution des datations radiocarbone pour les âges antérieurs à 20000 BP (Zilhao et d'Errico 2003 ; Fontugne 2004 ; Mellars 2004).

Ces datations placent, sans conteste, l'occupation du site dans une plage chronologique très ancienne pour le Gravettien.

\section{Industrie lithique}

\section{Matières premières lithiques}

Les matières premières lithiques présentent des caractéristiques qui semblent communes à l'ensemble des sites du Paléolithique supérieur de la partie centrale du Val d'Allier (Surmely et Pasty 2003), à savoir la domination, en proportion (90\% du total de l'industrie lithique, en poids), des silex locaux (silex intraformationnels tertiaires de l'Oligocène supérieur), l'existence de quelques rares silex régionaux (brèches filoniennes silicifiées du secteur de Madriat, Puyde-Dôme) et la présence de silex d'origine lointaine, avec, dans l'ordre décroissant d'importance, les silex de la craie du Turonien inférieur du Berry (fig. 2), les silex de l'Infralias (Hettangien) provenant très probablement du secteur de Saint-Jeanvrin (Cher ; étude en cours M. Piboule) et les calcarénites silicifiées du Turonien supérieur du secteur du Grand-Pressigny. Les occupants du site ont donc eu recours à des sources d'approvisionnement variées.

Malgré des stratégies d'exploitation bien distinctes, les trois grandes catégories de matières premières (silex crétacé du Turonien inférieur du Berry, silex tertiaire local et silex de I'Infralias de Saint-Jeanvrin) semblent avoir été utilisées de façon indifférenciée pour le façonnage des divers types d'outils. Tout juste peut-on observer une légère préférence des silex tertiaires pour la production d'armatures microlithiques et à l'inverse des silex turoniens pour les pièces esquillées. Bien évidemment, d'autres paramètres nous restent inconnus (utilisation de certaines pièces par certains types d'individus du groupe humain, valeur supérieure de certaines pièces, selon des critères que nous ne pouvons mettre en évidence...) et rendent une conclusion définitive impossible. Mais l'on peut 


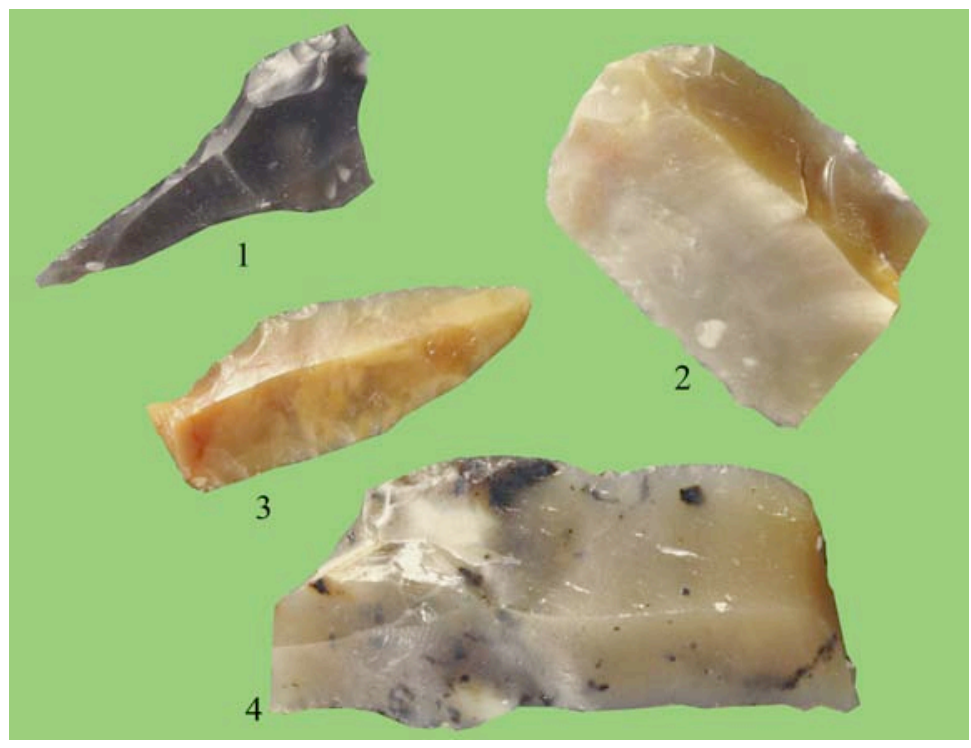

Figure 2 - Le Sire (Mirefleurs, 63) -

Gravettien ancien, niveau supérieur. Outils en silex de la craie du Turonien inférieur. 1 : pointe de la Font-Robert. 2 : grattoir sur lame. 3 : fléchette. 4 : lame retouchée. Photo F. Surmely.

Figure 2 - Le Sire (Mirefleurs, 63) - Early Gravettian, upper level. Lower Turonien chaulk flint tools. 1 : Font-Robert point. 2 : scraper on blade. 3 : projectile point. 4 : retouched blade. Photo F. Surmely.

dire que rien, selon les critères pris en compte, ne permet d'affirmer que les silex allochtones avaient une valeur fonctionnelle supérieure à celle des silex tertiaires locaux que les gravettiens avaient quasiment sous leurs pieds. La même étude conduite sur les corpus d'autres gisements du Paléolithique supérieur du Massif central (Le Blot, Le Pont-deLongues, Thônes, Le Cheix, Béraud, la Bade de Collandres...) aboutit au même constat (Surmely sous presse).

La justification de l'importation de silex d'origine lointaine nous semble donc à chercher en dehors du domaine purement technique, comme nous l'avions déjà supposé (Surmely et Pasty 2003). A l'évidence, ce recours à des matières premières allochtones, à la fois précieuses, belles et bien différenciées par la couleur des matériaux locaux, pourrait répondre à un désir de prestige social, en tous points comparable à celui qui présidait à l'utilisation d'éléments rares pour la parure (Vanhaeren et d'Errico 2003).

\section{Typotechnologie}

Les considérations qui suivent sont basées sur l'étude préliminaire du niveau supérieur, le niveau inférieur n'ayant été à ce jour reconnu que sur une assez faible surface. II faudra attendre les remontages et les études technologiques détaillées, en comparant les chaînes opératoires mises en œuvre sur les différents types de matières premières. Nous donnons donc ici un aperçu rapide des observations préliminaires.

De prime abord, on observe peu de différences entre les deux niveaux, ce qui confirme les indications données par les datations ${ }^{14} \mathrm{C}$ sur le rattachement des deux occupations à une même phase culturelle.
L'industrie lithique est caractérisée par la recherche d'un débitage laminaire et lamellaire de qualité (fig. 3), à partir de nucléus principalement unipolaires, mais aussi bipolaires, avec l'utilisation de percuteurs tendres pour le débitage des supports allongés. Les préparations se cantonnent en une abrasion de la corniche et les talons en éperon sont très rares.

Les plaquettes de silex tertiaire, apportées sur le site à l'état presque brut (fig. 4-1), sont travaillées par façonnage de crêtes sur la tranche et un débitage dans le sens du litage naturel de la roche siliceuse, avec beaucoup de lames à trois pans à section légèrement dissymétrique. Cela constitue un mode habituel, pour le Paléolithique supérieur, d'exploitation des plaquettes de silex tertiaires.

Le silex crétacé d'importation est arrivé sur le gisement à l'état de nucléus préformés, façonnés dans des rognons allongés et cylindriques, voire quelquefois sous forme de supports. Les lames à deux pans dominent.

II n' y a pas de chaîne opératoire de fabrication d'armatures à partir de chutes de burin du type du Raysse (Klaric 1999). Les lamelles semblent avoir été extraites de véritables nucléus à lamelles, dont certains exemplaires sont parvenus jusqu'à nous.

La question de la continuité éventuelle entre production de lames et de lamelles, n'a pas été éclaircie à ce jour.

Les outils sont représentés surtout par les armatures microlithiques ( $43 \%$ de l'outillage total), parmi lesquelles dominent largement les pointes à dos de type microgravette $^{6}$ (fig. 5) (Hays et Surmely 2005). II faut rappeler que le tamisage exhaustif à l'eau des sédiments, à l'aide de tamis

(6) La définition typologique de cet outil varie beaucoup d'un auteur à l'autre (voir note 8), ce qui est une gêne certaine pour les comparaisons. En ce qui nous concerne, et suivant en cela la définition donnée par P. Laurent et P.-Y. Demars, nous parlons de microgravette pour toute pointe microlithique à dos droit aménagée sur lamelle ou esquille lamellaire, présentant une retouche sur le bord opposé, inverse ou directe et partielle ou continue. A défaut de cette retouche sur le bord opposé, on parlera de pointe à dos simple. Les exemplaires non pointus sont rangés dans la catégorie des lamelles à dos, les fragments mésiaux dans celle des armatures microlithiques non identifiées. 

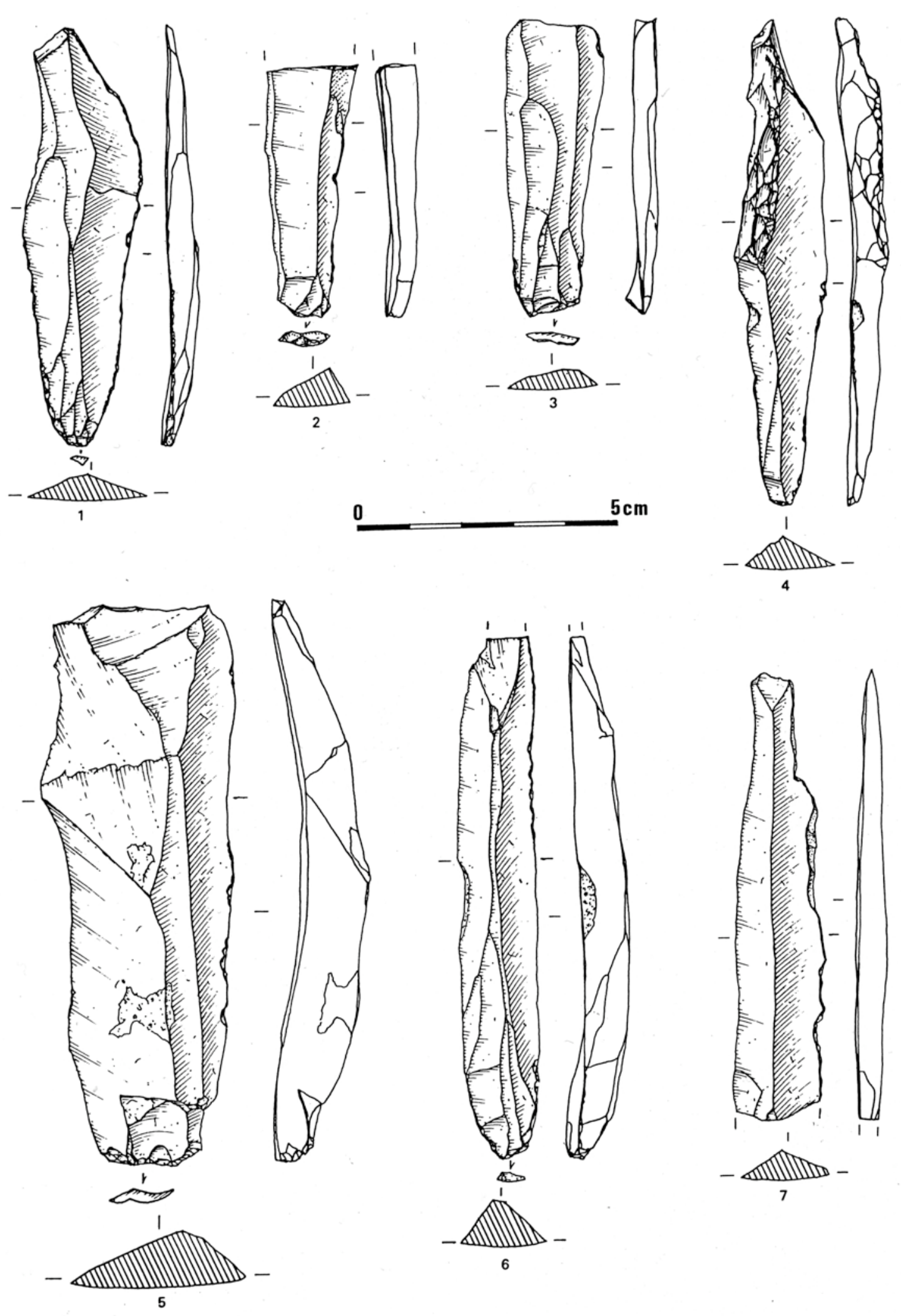

Figure 3 - Le Sire (Mirefleurs, 63) - Gravettien ancien, niveau supérieur. Lames en silex tertiaire local. Dessins Ph. Alix. Figure 3 - Le Sire (Mirefleurs, 63) - Early Gravettien, higher level. Blades of local tertiary flint. Drawings Ph. Alix. 

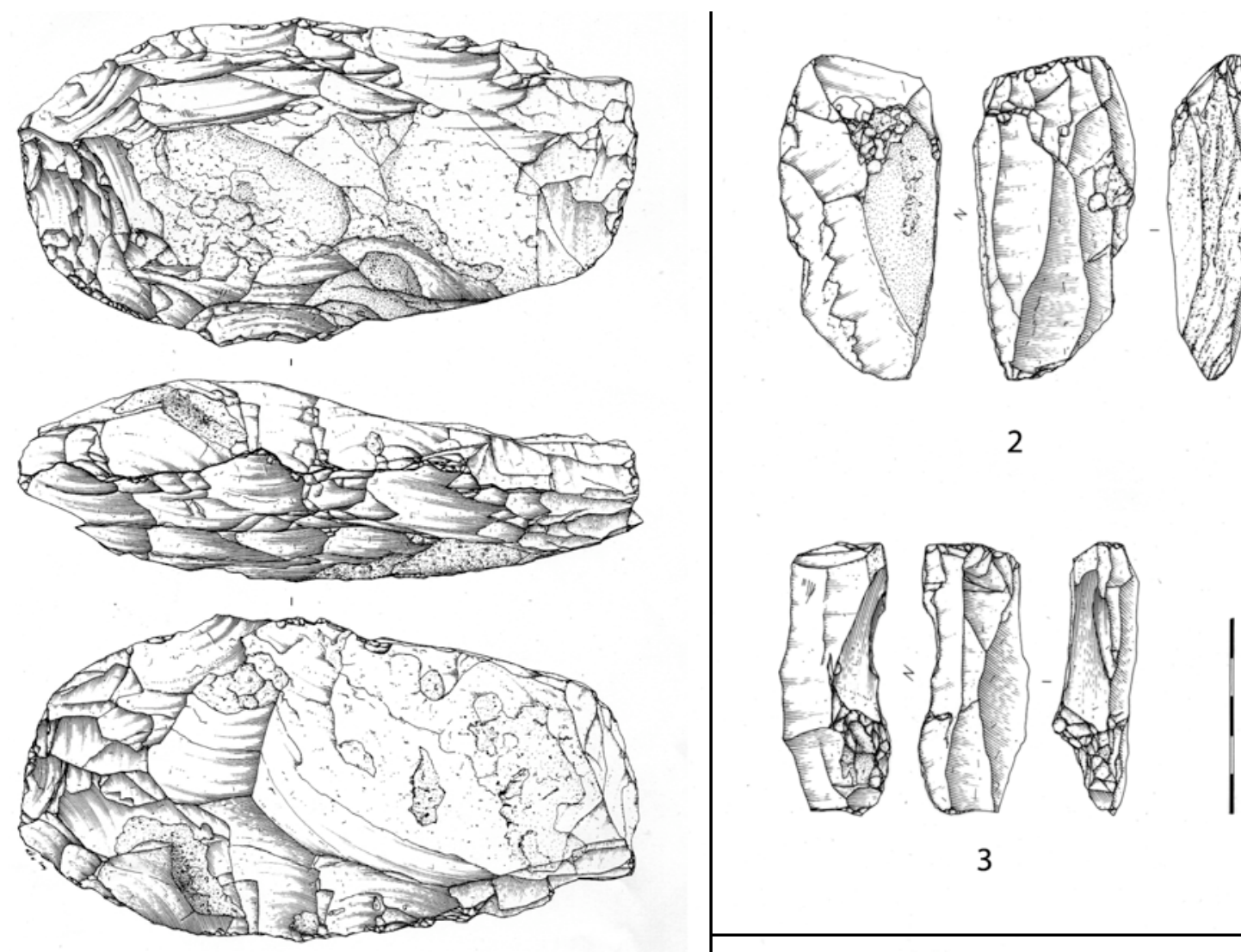

3
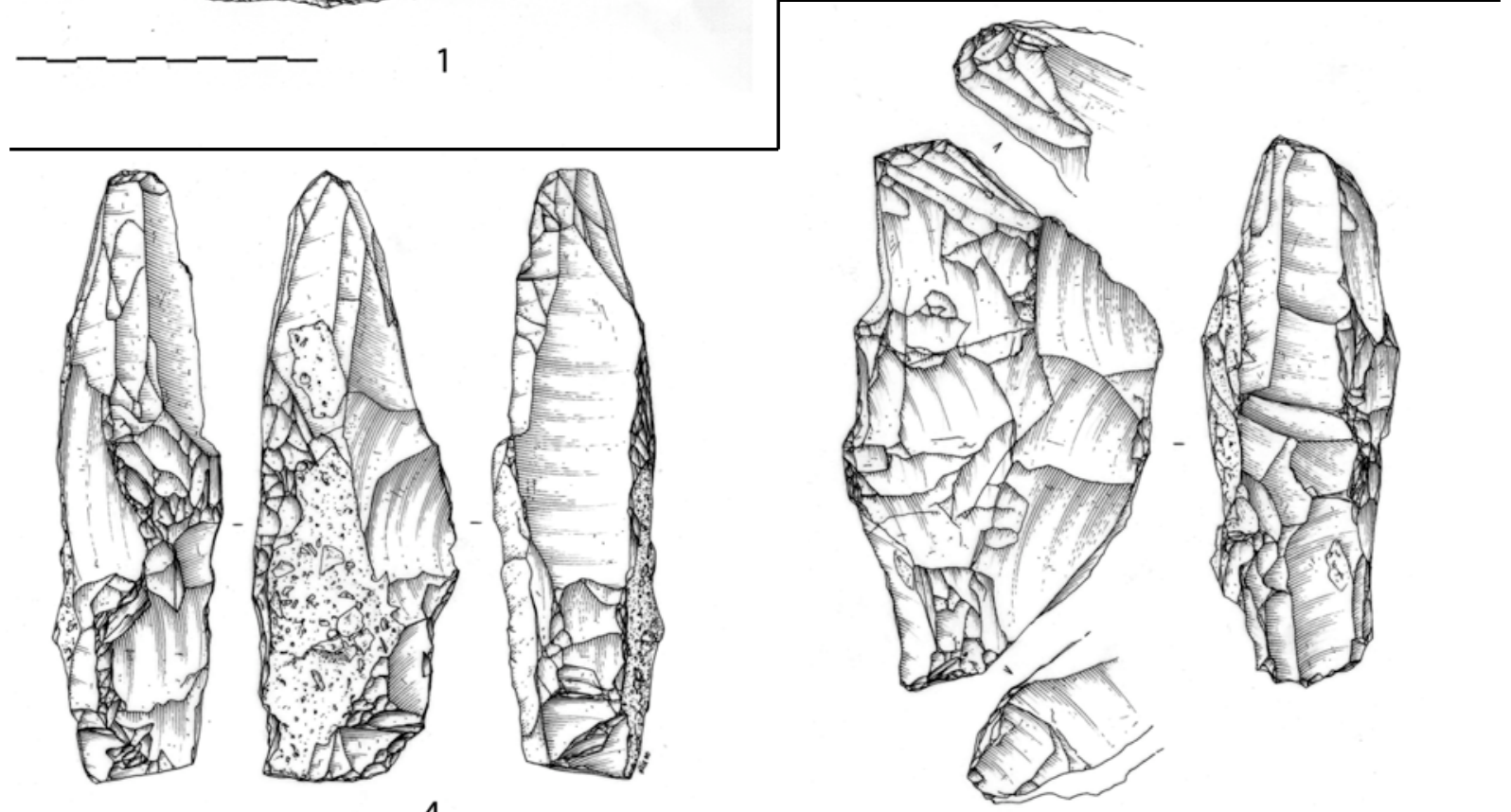

4

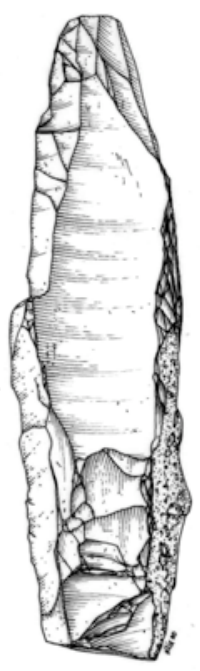

Figure 4 - Le Sire (Mirefleurs, 63) - Gravettien ancien, niveau supérieur : 1, 4 et 5 - niveau inférieur : 2 et 3. Préforme $\left(n^{\circ} 1\right)$ et nucléus en silex tertiaire local. Dessins Ph. Alix.

Figure 4 - Le Sire (Mirefleurs, 63) - Early Gravettian, higner level : 1, 4 and 5 - lower level : 2 and 3. Preform ( $\left.n^{\circ} 1\right)$ and core of local tertiary flint. Drawings Ph. Alix. 


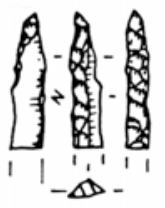

1

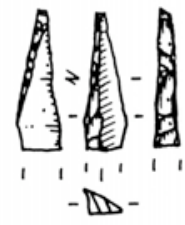

2

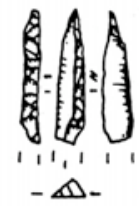

3

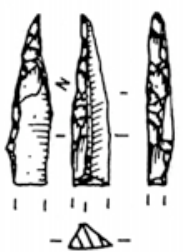

4

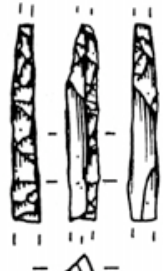

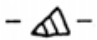

Figure 5 - Le Sire (Mirefleurs, 63) - Gravettien ancien, niveau supérieur. Microgravettes. Dessins Ph. Alix.

Figure 5 - Le Sire (Mirefleurs, 63) - Early Gravettian, higher level. Microgravettes. Drawings Ph. Alix.

à maille fine, a permis une collecte exhaustive des microlithes. Les lames retouchées arrivent en seconde position (22\%), suivies par les grattoirs (13\%), les burins (9\%) et les pièces esquillées (5\%) (fig. 6). On trouve enfin, en petit nombre, des perçoirs et becs, des outils mixtes, des éclats retouchés. II est à noter la découverte de plusieurs lames appointées typologiquement très proches de celles du Protomagdalénien (cf. infra). Les pointes de La Gravette sont assez peu abondantes (3\%). On constate également la présence d'une pointe de La Font-Robert et d'une fléchette (fig. 2-1 et 2-3). Dans le niveau inférieur, les armatures semblent moins abondantes, avec un plus grand nombre de burins, dont la proportion atteint celle des grattoirs.

L'outillage lithique du niveau supérieur du Sire présente ainsi un profil typologique tout à fait comparable à celui des autres gisements du Gravettien ancien d'Europe et notamment du site de Geissenklosterle (Hahn 1993), avec comme critères caractéristiques l'abondance des microgravettes, la présence beaucoup plus discrète des pièces esquillées, FontRobert, fléchettes et l'absence totale de burins de Noailles.

Plus près de nous, le site se rapproche également de celui de la Vigne Brun (Loire ; Digan 2001) et du niveau 5 de l'Abri Pataud (Leoz 2001).

Une étude tracéologique a été conduite sur les microgravettes, afin de préciser leur rôle fonctionnel. Les arguments ont été développés dans un autre article (Hays et Surmely 2005). Malgré le petit nombre de pièces étudiées (37), il apparaît que toutes les microgravettes portant des traces d'utilisation ont été utilisées comme pointes de projectile. Leur poids et surtout leurs dimensions ne peuvent correspondre qu'à un projectile de type flèche d'arc, ce qui recule l'utilisation de cette arme dans le temps, comme l'avaient supposé plusieurs chercheurs.

\section{Archéozoologie}

Les restes de faune sont très abondants, puisqu'ils représentent près de $70 \%$ des vestiges cotés lors de la fouille. Comme nous l'avons dit, ils forment par endroits de véritables amas.
Le Cheval correspond à l'espèce dominante dans l'ensemble des secteurs fouillés. Sa fréquence varie cependant d'un niveau à l'autre. II domine très largement (plus de $90 \%$ ) dans le niveau supérieur. II est moins bien représenté dans le niveau inférieur (60\% environ), au profit du Renne. L'abondance des restes de loups (10\%) qui proviennent d'au moins trois individus, est également remarquable dans cette dernière couche.

On note l'absence complète de restes de petits animaux, terrestres et aquatiques, ce qui peut être imputé, en première analyse, à un problème de conservation.

En ce qui concerne les profils squelettiques, les nombres minimums d'éléments n'ont pas encore été calculés. Cependant, d'après D. Grayson et de C. Frey (en prép.), il semble que les profils squelettiques obtenus sur la base des nombres minimums d'éléments (NME) soient très proches de ceux établis à partir des nombre de restes NRD). En procédant de la sorte, il apparaît, dans les deux niveaux considérés, une nette sous-représentation du squelette axial post-crânien et des os courts. Les éléments les plus abondants sont les métapodes et, dans une moindre mesure, le tibia. La faible représentation des os courts pourrait résulter du mode de calcul utilisé. En effet, contrairement au NME, le NRD ne permet pas de prendre en compte des taux de fragmentation différentiels selon les ossements or, les os courts sont souvent complets alors que les os longs sont retrouvés sous forme de fragments. Par ailleurs, les attaques corrosives observées sur certaines pièces pourraient être à l'origine de la disparition d'une partie de ces éléments qui sont riches en tissu spongieux donc plus susceptibles de destruction. La présence de vertèbres particulièrement bien conservées ne permet pas pour autant d'exclure un problème de conservation différentielle : certaines pièces pourraient en effet avoir subi une forte attaque corrosive qui serait à l'origine de leur disparition. En ce qui concerne les os des membres, les éléments riches en viande, scapula, humérus, os coxal et fémur, sont peu abondants.

Les données relatives au traitement des carcasses sont limitées en raison de la mauvaise conservation des sur- 


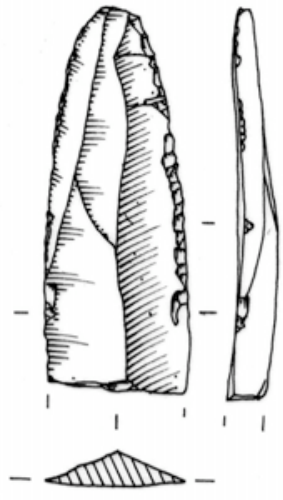

1
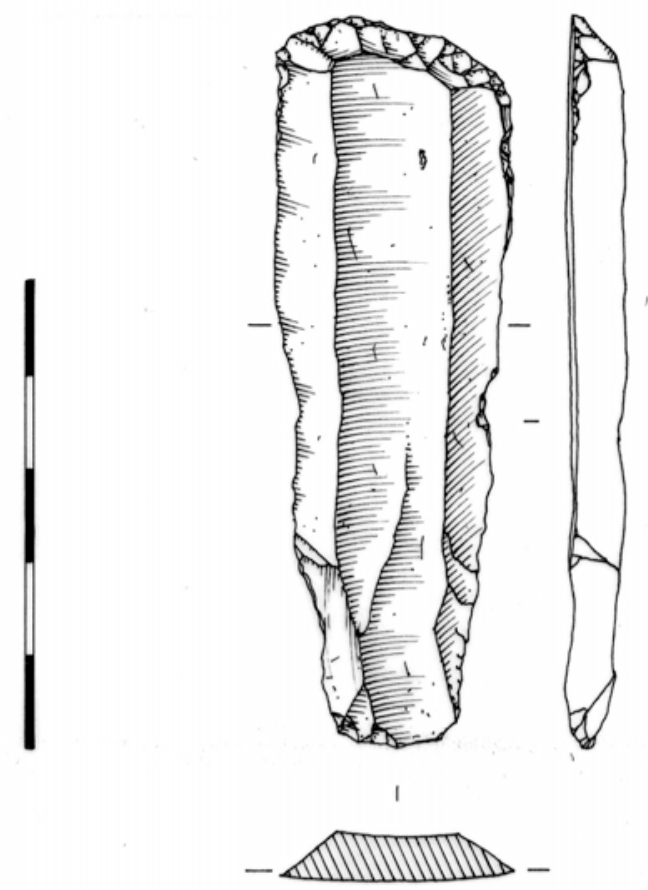

2
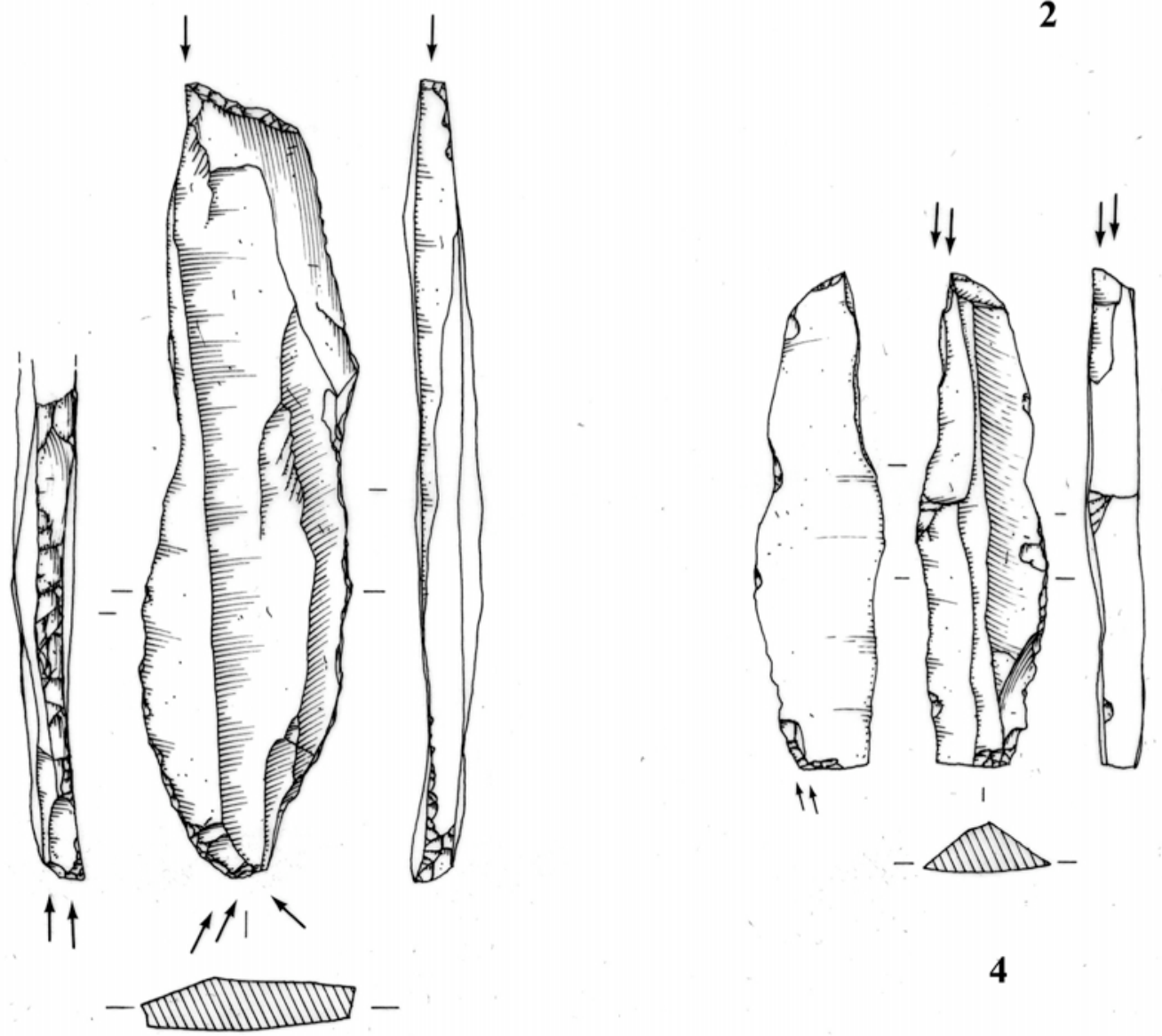

Figure 6 - Le Sire (Mirefleurs, 63) - Gravettien ancien, niveau inférieur. Outils de fonds commun. Dessins Ph. Alix. Figure 6 - Le Sire (Mirefleurs, 63) - Early Gravettian, lower level. Commonly found tools. Drawings Ph. Alix. 
faces osseuses qui empêche la lecture des stries de boucherie. La présence de nombreux os longs entiers montre cependant une exploitation peu intensive des carcasses.

Malgré la très grande abondance des ossements brûlés, I'hypothèse d'une utilisation de l'os comme combustible reste à confirmer par une étude détaillée des résidus osseux issus des combustions (Costamagno et al. 2005 ; Théry-Parisot et Costamagno sous presse).

\section{Industrie osseuse, objets d'art et de parure}

Le site se caractérise par l'absence totale d'industrie osseuse. Cette caractéristique pourrait s'expliquer, en première analyse, par la domination du Cheval dans la faune chassée, ce qui aurait induit une faible disponibilité en bois de cervidés et le remplacement de l'industrie osseuse habituelle par des pièces lithiques.

Les objets d'art et de parure sont rares et se limitent à un crache de cerf perforé (étude en cours F. d'Ericco), un fragment de pendeloque circulaire doté d'incisions périphériques, un morceau d'hématite présentant des stries et un petit fragment osseux portant des stries volontaires. II est à noter enfin que des poches de sédiment ocré ont été retrouvées dans les deux niveaux.

\section{Caractérisation du site}

Ces résultats permettent de formuler un certain nombre d'hypothèses provisoires quant à la fonction du site et sa place au sein du cycle saisonnier des groupes humains ayant occupés le Sire. Le choix de l'emplacement du campement, sur le versant d'une colline offrant une large vue sur la vallée de l'Allier, ne semble pas fortuit. L'hypothèse d'un site d'abattage peut être exclu en raison de l'abondance de l'outillage lithique, mais également de la mise en évidence d'importantes activités de boucherie sur les carcasses. Un site de boucherie primaire au sens strict peut également être rejeté. En effet, la présence de foyers associés à des os brûlés et la mise en évidence d'une consommation sur le gisement d'au moins une partie des ressources fournies par les animaux abattus témoignent d'un site occupé pendant un certain laps de temps ce qui va à l'encontre de la définition généralement retenue pour un site de boucherie stricto sensu.

Le gisement pourrait donc correspondre soit à une halte de chasse occupée durant quelques jours dans le but de traiter les carcasses de chevaux abattus à proximité du site et de transporter les ressources alimentaires (ou d'autres ressources comme la peau) ainsi obtenues sur un autre gisement, soit à un camp résidentiel occupé durant un laps de temps relativement long où l'ensemble des ressources alimentaires était consommé sur place. L'introduction d'ossements qui ne sont pas exploités ensuite (présence de nombreux os longs entiers) témoigne de sites d'abattage relativement proches du gisement. Si cette proximité des sites d'abattage va dans le sens d'une halte de chasse, elle ne permet pas d'exclure l'hypothèse d'un camp résidentiel dont l'emplacement aurait été choisi en raison, justement, de son faible éloignement des territoires de chasse.

L'absence des parties nutritivement les plus riches sur le site est un argument fort en faveur d'une halte de chasse. L'abondance des armatures semble également étayer l'hypothèse d'occupations de durées relativement courtes, ciblées sur la chasse aux chevaux : l'étendue du gisement témoignerait alors de présences récurrentes au fil des ans. Cependant, la surface fouillée à ce jour, par rapport à l'extension totale du site ne permet pas de rendre compte d'une éventuelle segmentation des activités dans l'espace. Ainsi, les ossements charnus pourraient être présents sur le site mais dans une zone non fouillée, ce qui serait alors l'indication d'un camp résidentiel et non plus d'une halte de chasse.

Les datations placent sans conteste l'occupation du site dans une plage chronologique très ancienne pour le Gravettien. Elles figurent même parmi les plus anciennes obtenues en Europe pour le Gravettien.

Cela amène à des conclusions de portée extra-régionale. Le Gravettien ne peut plus être défini comme une culture née en Europe orientale ou centrale. L'hypothèse d'un foyer oriental du Gravettien, voire d'un " couloir $d u$ Danube ", doit être réfutée ou, du moins, discutée selon des critères autres que ceux du carbone 14 (compte tenu des incertitudes de la calibration évoquées plus haut). II faut aussi réviser l'idée selon laquelle le Gravettien serait apparu vers 28000 BP (Djinjian et al. 1999).

Même si le gisement du Sire peut apparaître comme isolé dans cette plage chronologique à ce jour, il faut prendre en considération, d'abord que les gisements du Gravettien ancien ne sont pas nombreux en France et que les principales occupations pouvant s'y raccorder (La Vigne Brun ; Abri Pataud, couche 5 ; certains niveaux de Solutré) ont livré des dates paraissant douteuses ${ }^{7}$ qui demanderaient à être vérifiées par de nouvelles mesures par AMS.

Par ailleurs, ces datations sont très proches de celles obtenues pour les grands sanctuaires artistiques de la grotte Chauvet (France) et du Hölhenstein-Stadel (niv. IV), du Vogelherd et de Geissenklosterle II (Allemagne ; Hahn

(7) Pour la Vigne-Brun : $18520 \pm 500$ BP (Ly-391); $24900 \pm 2000$ BP (Ly-391); $19500 \pm 480$ (Ly-2151); $20840 \pm 390$; $23450 \pm 690$ (Ly-2637) ; $21580 \pm 600$ (Ly-2368); $23230 \pm 760$ (Ly-2639) ; $23500 \pm 1000$ (Ly-2640). Données extraites de la base de données BANADORA-Université de Lyon 1.

Quant aux datations ${ }^{14} \mathrm{C}$ réalisées sur l'ensemble du gisement de l'Abri Pataud, recensées par la base BANADORA du centre de radiocarbone de Lyon 1, sont en nombre bien supérieur à celui donné dans la publication de H.-M. Bricker (1995). Leur variabilité est également bien plus grande et l'ensemble apparaît peu fiable. 
1993 ; Djinjian et al. 1999). L'attribution traditionnelle de ces sites à l'Aurignacien II, qui repose essentiellement sur les données radiocarbones, serait peut-être à revoir, notamment pour la grotte Chauvet.

\section{LE GRAVETTIEN MOYEN OU RÉCENT : LES NIVEAUX 37 À 52 DU BLOT (CERZAT, HAUTE-LOIRE)}

Le site du Blot, sur la commune de Cerzat (Haute-Loire), est situé au pied d'un grand escarpement basaltique, en bordure de la rivière Allier (fig. 7), à une altitude de $630 \mathrm{~m}$ (Virmont 1981 ; Bosselin 1992). Découvert en 1934, il fut sondé par J. Combier en 1956, puis fouillé de 1964 à 1984 par H. Delporte, assisté de J.-P. Daugas et J. Virmont. Trois locus, baptisés " chantiers 1, 2 et 3 » ont fait l'objet de fouilles. Le remplissage, caractérisé par des mélanges d'éléments détritiques de toutes tailles issus de l'altération de la coulée basaltique sus-jacente, d'apports alluvionnaires dus à des crues de la rivière et de colluvions argileuses (Marguerie 1982 ; Bosselin 1992), est d'une grande complexité, rendant le découpage stratigraphique d'une rare difficulté. La base des niveaux archéologiques n'a pas été atteinte, les fouilles ayant été stoppées par la nappe phréatique.

II faut souligner la qualité des travaux de terrain, avec un relevé minutieux des vestiges et un tamisage à l'eau des sédiments, qui explique en grande partie l'abondance des microlithes.

Le Gravettien et le Protomagdalénien ont été reconnus dans le « chantier 3 ».

Le Gravettien est représenté par les couches 37 à 52 du chantier 3, correspondant " à une succession d'occupations restreintes et discontinues, au nombre minimum de 7 à 8 » (Virmont 1981). D. Buisson parle de « quatre couches majeures regroupant la vingtaine de niveaux reconnus à la fouille ", même si son étude est basée ensuite sur la prise en considération de trois "ensembles " (Buisson 1991). Enfin J.-P. Daugas a proposé un schéma de deux grandes phases d'occupations, séparées par un épisode stérile, composées d'une part, des couches 37 à 40 et 42 à 52 , d'autre part. Le niveau 39 est de loin le plus riche, puisqu'il regroupe la moitié des outils découverts dans toute la séquence périgordienne.

Ces chercheurs s'accordent à souligner la relative pauvreté en vestiges des différents niveaux et leur ressemblance, ce qui impliquerait des occupations assez brèves et proches dans le temps.

La couche 39 avait fait l'objet de deux datations ${ }^{14} \mathrm{C}$, réalisées par la méthode classique : $21700 \mathrm{BP} \pm 1200$ (Ly-564) et $21500 \pm 700$ (Ly-565) (Virmont 1981). Tout récemment, de nouvelles datations ont été effectuées par AMS : $24640 \pm 120$ (GRA-17336) pour le niveau 39, $22210 \pm 150$ (GRA-17217) pour le niveau 42 et $24610 \pm 200$ (GRA-17218) pour le niveau 48 (Klaric 2003). Ces nouvelles mesures donnent un âge plus ancien à la séquence malgré les incertitudes liées à la calibration imprécise pour cette période.

Sur le plan des matières premières, aucune étude détaillée n'a été réalisée à ce jour. Les différents chercheurs ayant examiné les séries témoignent de la présence de silex allochtones, mais sans en donner les proportions. Cellesci semblent plus réduites que dans les niveaux protomagdaléniens. J. Virmont mentionne aussi la présence de silex tertiaire de Limagne.

Du point de vue typologique, il faut se référer aux études de D. Buisson et B. Bosselin. 455 outils ont été décomptés sur l'ensemble des niveaux, dont 242 pour les seuls niveaux 39. Les armatures microlithiques représentent $77 \%$ avec $50 \%$

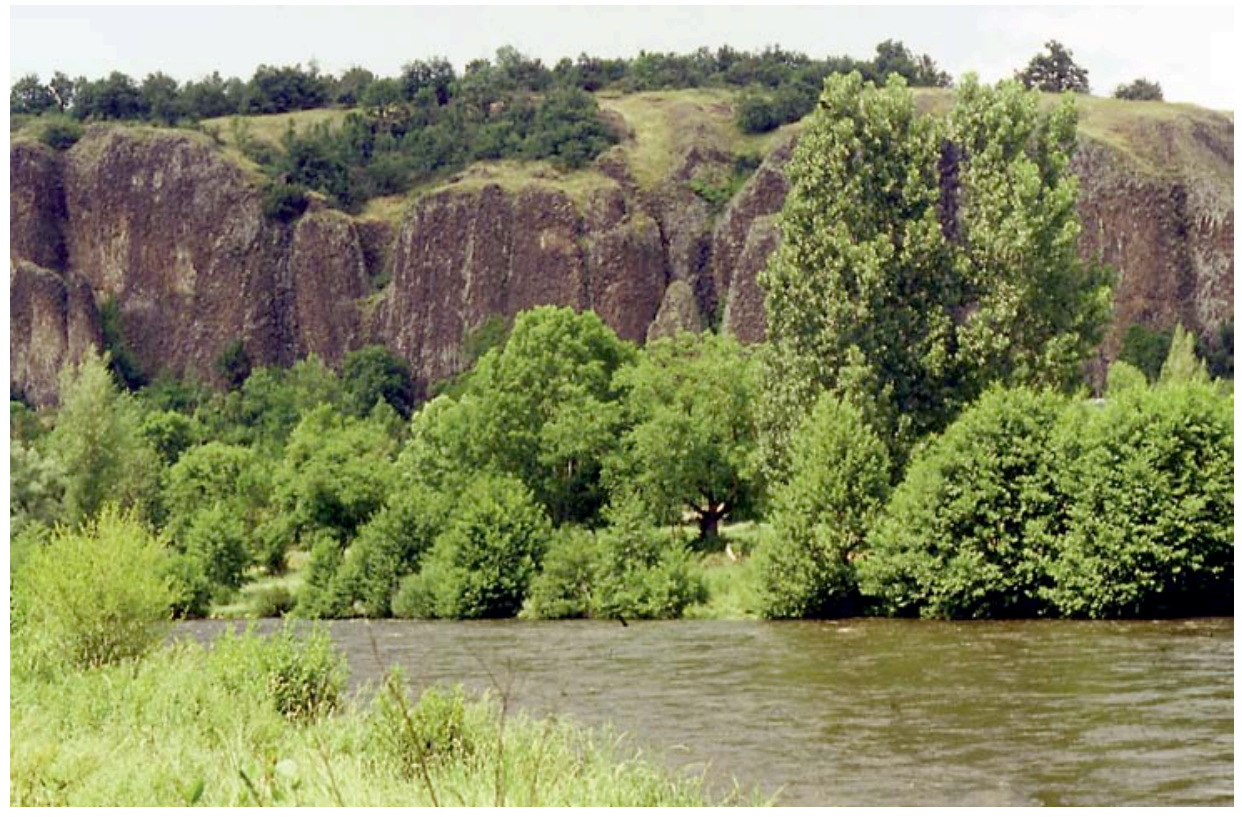

Figure 7 - Le Blot (Cerzat, 43) - Vue générale du site. Photo F. Surmely.

Figure 7 - Le Blot (Cerzat, 43) - General view of the site. Photo F. Surmely. 
de lamelles à dos et $27 \%$ de microgravettes ${ }^{8}$ (Bosselin 1992). Le second groupe est celui des burins (15,4\%) dont certains exemplaires rappellent les burins de Noailles. Les grattoirs sont presque totalement absents. Des variations s'observent selon les niveaux, avec une tendance allant à la diminution des microlithes du bas vers le haut de la série.

L'étude typotechnologique a été réalisée récemment par $L$. Klaric qui a identifié une production de lamelles à partir de lames épaisses. Laure Fontana a étudié la faune, les résultats restant encore inédits. L'outillage osseux est très réduit. Les objets de parure se réduisent à quatre perles et pendeloques en ivoire et en pierre (c. 39) et un galet orné de figurations animales.

Tous les auteurs attribuent, avec prudence, l'intégralité de la séquence à une phase récente du Gravettien. Les arguments présentés sont les suivants : premières datations ${ }^{14} \mathrm{C}$ et présence de lames appointées évoquant le Protomagdalénien sus-jacent (Virmont 1981 ; Buisson 1991), absence de burins du Raysse et présence de lamesnucléus (Klaric 2003). Nous avons vu que la fiabilité des premières dates a été mise en doute par la réalisation de mesures récentes par AMS, qui indiqueraient des âges plus anciens. Les points communs entre les deux séquences gravettiennes et protomagdaléniennes, à savoir l'abondance des microlithes et la présence de lames appointées, peuvent s'expliquer d'une part, en partie au moins, par la mise en œuvre d'un tamisage à l'eau et d'autre part, en raison de problèmes stratigraphiques.

\section{UN SITE GRAVETTIEN PROBABLE : LE SITE DE TÂTEVIN (CHANTEUGES, HAUTE-LOIRE)}

La grotte de Tâtevin, située sur la commune de Chanteuges (Haute-Loire) s'ouvre sur le flanc sud-ouest d'un long escarpement basaltique orienté sud-ouest (Virmont 1981). Elle domine l'Allier d'environ $30 \mathrm{~m}$. Découverte et sondée anciennement, le site a fait l'objet de deux campagnes de fouilles en 1971 et 1973, sous la conduite de J. Virmont. L'industrie lithique se caractérise par l'abondance des burins (plus d'un tiers de l'outillage), la présence des grattoirs (15\%) et des pièces à dos, parmi lesquelles figure une pointe de La Gravette. Malgré les différences par rapport aux séries du Blot, J. Virmont attribue le mobilier au Gravettien. Toutefois, ce diagnostic a été contesté par D. Buisson, qui penchait plutôt pour un rattachement au Protomagdalénien (Buisson 1991).

\section{LE PROTOMAGDALÉNIEN DU GISEMENT DU BLOT}

Le Protomagdalénien est une culture encore peu connue et sa présence en Auvergne mérite assurément un développement. Caractérisé à Laugerie-Haute (Peyrony 1938), le Protomagdalénien (ou Périgordien VII, cf. infra) se distingue aussi des autres cultures du Paléolithique supérieur par sa rareté. II n'a en effet été identifié clairement à ce jour que dans quatre gisements français : Laugerie-Haute-Est c. F, 36 et 38, LesEyzies-de-Tayac, Dordogne ; Peyrony 1938 ; Bordes 1978 ; Bordes et Sonneville-Bordes 1966 ; SonnevilleBordes 1960 ; Demars 1994), Pataud c. 2 (Bosselin 1992 ; Clay 1995 ; Kong-Cho 1997), Le Blot (Cerzat, Haute-Loire ; Delporte 1976 ; Bosselin 1992 et 1997) et Les Peyrugues (Cabrerets, Lot ; fouille M. Allard). Sa datation semble à placer autour de $20000 \mathrm{BP}$, d'après les datations ${ }^{14} \mathrm{C}$.

\section{Datation}

Aucune datation n'est disponible à ce jour pour les niveaux protomagdaléniens du Blot.

\section{Stratigraphie et organisation spatiale}

Le Protomagdalénien se rencontre dans les niveaux 22 à 34 du «Chantier 3 ». Comme pour le Gravettien, le découpage stratigraphique est d'une grande complexité, renforcé en cela par un changement de dénomination des couches en cours de fouille. La séquence protomagdalénienne est séparée des niveaux gravettiens d'une part et badegouliens d'autre part par des couches stériles, ce qui n'exclut toutefois pas des mélanges dus à la nature hétérogène du remplissage de l'abri. J.-P. Daugas a proposé récemment un découpage de la séquence en trois phases d'occupation : phase 2 inf., phase 2 sup. et phase 3 . Le nombre exact de niveaux d'occupation reste indéterminé.

Un aménagement de gros blocs issus de l'altération du surplomb a pu être mis en évidence dans la phase 2 sup. $\mathrm{H}$. Delporte et J. Virmont ont cru voir une « cabane » de 8 à 10 $\mathrm{m}$ de long pour quatre de large. B. Bosselin semble plus prudent quant à l'interprétation (Bosselin 1992). Les « structures » présumées ont fait l'objet de multiples réaménagements au cours du temps, jusqu'au début de la « phase » 3.

\section{Archéozoologie}

Les vestiges organiques d'origine animale ont été étudiés récemment par F.-X. Chauvière et L. Fontana (Chauvière et Fontana sous presse 1 et 2). Le Renne constitue l'essentiel de la faune chassée ( $88 \%$ des 2011 restes de faune). Les animaux semblent avoir été tous abattus entre les mois de mai et de septembre. Les carcasses ont été apportées entières sur le site. Aucune différence significative n'a pu être observée entre les différents niveaux. vindtsept objets d'art et de parure, dont des poinçons et des perles en ivoire, ont été découverts mais aucun élément de projectile en matière animale n'a été retrouvé.

(8) Selon les critères typologiques retenus par B. Bosselin : armature microlithique présentant un dos à retouches croisées et des retouches d'aménagement de la pointe ou d'amincissement de la base (Bosselin 1992). II est à noter que ce chercheur range tous les fragments mésiaux d'armatures dans la catégorie des lamelles à dos, alors qu'il nous paraît plus logique (cf. note 6) de les classer comme des armatures de type indéterminé. 


\section{Industrie lithique}

Elle n'est pas très abondante, compte tenu de l'existence de nombreux niveaux.

\section{Matières premières : origines et gestion}

Les séries protomagdaléniennes se caractérisent par la prépondérance quasi-exclusive des matières premières allochtones. Ce constat avait été effectué par A. Masson (Masson 1981, 1983 et 1986). Nos propres travaux, sur la base des études microfaciologiques de J.-C. Foucher (Université de Reims) et P. Barrier (IGAL) ont permis d'en confirmer l'exactitude, parfois de l'affiner et de le compléter par un décompte effectué sur la base du poids et non seulement du nombre de pièces.

Le type majoritaire, (52 à $60 \%$ des matières premières, en poids, selon les niveaux) est représenté par les silex bien connus des calcarénites du Turonien supérieur du secteur du Grand-Pressigny (type 23 d'A. Masson ; Giot et al. 1986 ; Aubry 1991). On retrouve au Blot les variations de couleur et de texture connues sur les gîtes primaires (fig. 8 à 11).

Le second type, en importance (27 à $43 \%$ du poids total), est formé par les silex de la craie du Turonien inférieur. Deux sous-variétés se rencontrent : la plus abondante est de teinte blond-gris clair (type 07 d'A. Masson ; Masson 1981). Mais il existe aussi une sous-variété de teinte grise ou noire, également translucide Masson 1981 ; Surmely et al. 1998). Cette attribution a été confirmée par un examen en lame mince effectué par J.-C. Foucher et nous-mêmes, sur la base de l'identification des dinoflagellés et acritaches (Foucher 1979 ; Surmely et al. 2002). L'origine géographique est évidemment plus difficile à déterminer, ce type de silex étant présent sur la bordure sud et est du Bassin parisien et notamment en Berry (canton de SaintAignan, Loir-et-Cher), en Champagne (secteur d'Estissac, Aube) et en Gâtinais (Loiret), le Berry étant le plus proche du gisement du Blot. Des études géochimiques ont permis de confirmer ce rapprochement, sans toutefois apporter de preuves formelles (Gervais 2001). Des études complémentaires sont en cours par l'un d'entre nous (FS).

Un ensemble de matériaux qui représente 5 à $13 \%$ de l'approvisionnement regroupe des silex issus de formations marines du Crétacé, dont l'origine est à chercher sans doute dans le sud du Bassin parisien et, dans une très faible proportion, un matériau de teinte orangé à marron, souvent bariolée, qui évoque le silex de l'Infralias du secteur de Saint-Jeanvrin (Cher).

Enfin, il faut signaler la présence, dans le niveau 2 supérieur seulement, de quelques pièces en silex tertiaire, de couleur

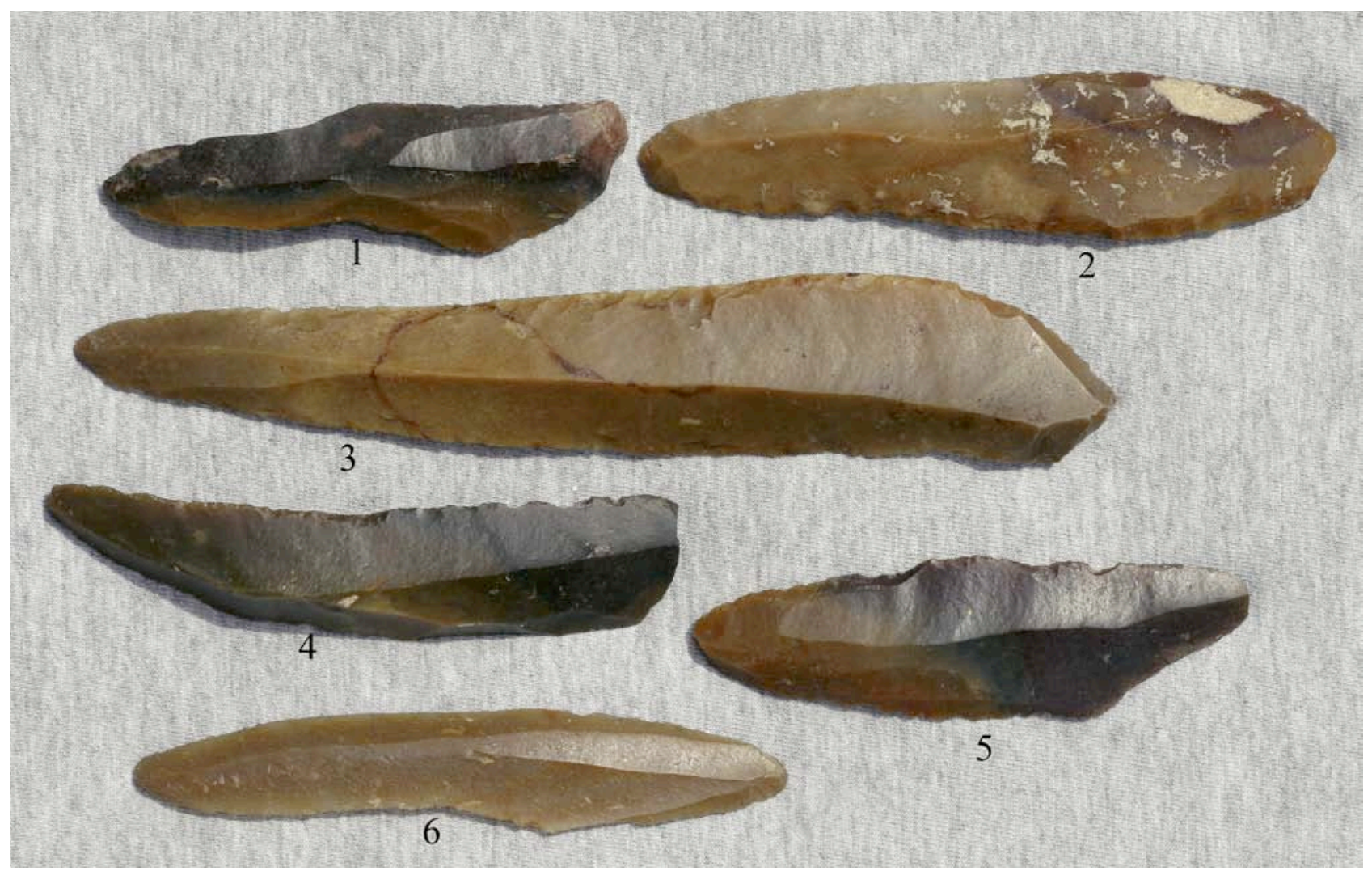

Figure 8 - Le Blot - Protomagdalénien. Lames en silex du Grand-Pressigny. Photo F. Surmely.

Figure 8 - Le Blot - Protomagdalenian. Blades of Grand-Pressigny flint. Photo F. Surmely. 


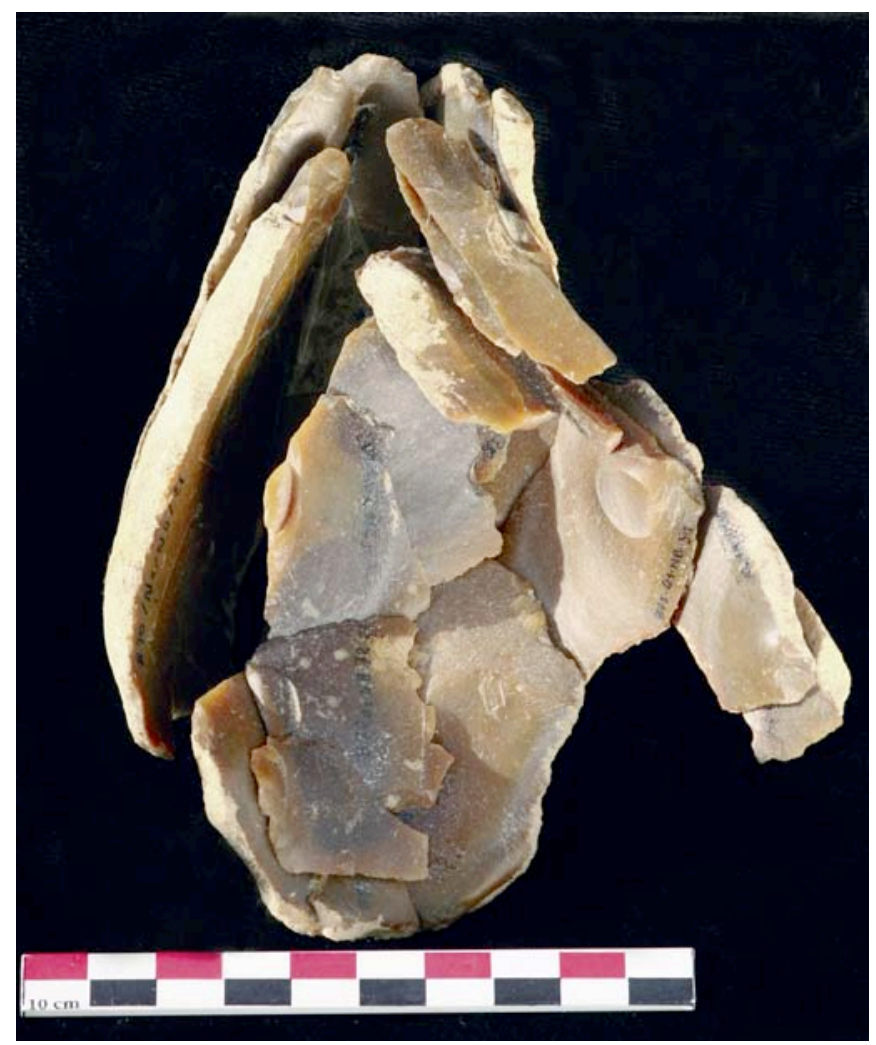

Figure 9 - Le Blot - Protomagdalénien. Remontage (31 pièces). Exploitation, en unipolaire, d'un bloc de silex brut de type silex pressignien, zoné, initialement piriforme. Production de 5 lames corticales, dont 2 à talon cortical, à partir de l'extrémité pointue du bloc, puis décorticage des flancs. Nucléus disparu. Remontage J.-F. Pasty, photo F. Surmely.

Figure 9 - Le Blot - Protomagdalenian. Refitting (31 pieces). Unipolar exploitation of a rough block of pressignian flint, initial periform zone. Production of 5 cortical blades, including 2 with a cortical butt, initiating from the pointed end of the block, then removal from the sides. Core not present. Refitting J.-F. Pasty, photo F. Surmely.

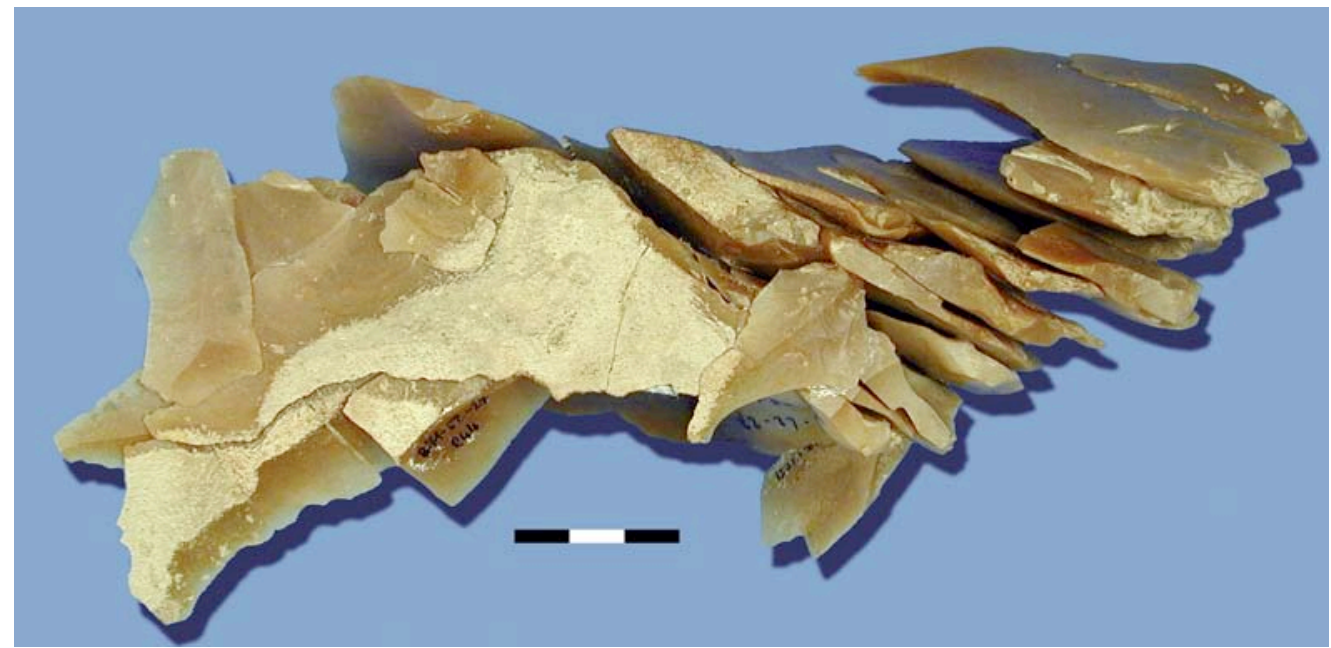

Figure 10 - Le Blot - Protomagdalénien. Remontage (63 pièces) d'un nucléus en silex pressignien, montrant la succession des tablettes et éclats de ravivage, liée au débitage de lames à talons en éperon. Remontage et photo J.-F. Pasty.

Figure 10 - Le Blot - Protomagdalenian. Refitting (63 pieces) of a pressignian flint core, showing the succession of tablets and reduction flakes, related to the detachment of blades with spur shaped butts. Refitting and photo J.-F. Pasty. 


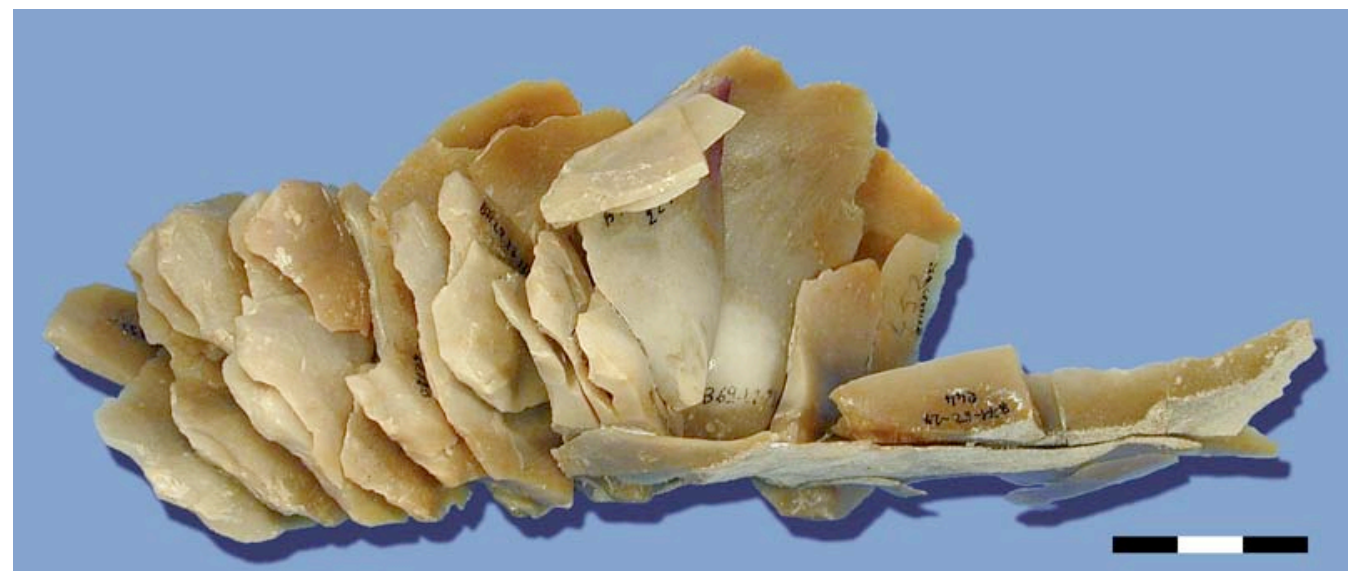

Figure 11 - Le Blot - Protomagdalénien. Autre vue du remontage de 63 pièces. Remontage et photo J.-F. Pasty.

Figure 11 - Le Blot -Protomagdalenian. Refitting, additional view of 63 pieces. Refitting and photo J.-F. Pasty.

gris clair et à texture homogène. Sur la base d'analyses fondées notamment sur la géochimie, N. Dufresne a pu montrer que ces silex tertiaires ne correspondent pas aux matériaux d'aspect semblable que l'on peut trouver dans les bassins sédimentaires tertiaires d'Auvergne (Dufresne 1999). Comme l'avait déjà supposé $A$. Masson, il s'agit très vraisemblablement de matériaux provenant de régions extérieures. Une étude géochimique comparative (Giez 2001) n'a pas permis de faire le rapprochement avec les silex tertiaires du sud (Irribaria 1992) et du centre du Bassin parisien (Mauger 1985). L'origine géographique de ces matériaux, qui sont beaucoup plus abondants dans les niveaux magdaléniens du même site (étude en cours, F. Surmely), reste donc à déterminer. II n'y a pas de silex tertiaire de Limagne, malgré ce qui a pu être indiqué parfois (Bosselin 1992).

En conclusion, il faut donc souligner que la totalité des matériaux utilisés par les protomagdaléniens du Blot sont, sans contestation possible, d'origine étrangère à la région. Cela est d'ailleurs confirmé par la gestion particulière de l'approvisionnement, qui est typique de roches d'origine lointaine. Ce recours exclusif aux silex allochtones s'explique assurément par l'absence de bons matériaux locaux (Surmely 1998 ; recherches en cours de P. Fernandes et J.-P. Raynal). II est à noter enfin que les silex tertiaires de Limagne sont totalement absents des séries protomagdaléniennes du Blot.

Il est difficile d'établir des comparaisons interrégionales, vu le très faible nombre de sites de la première moitié du paléolithique supérieur. Observons toutefois que le gisement du Blot se singularise par la très forte proportion des silex attribués au secteur du Grand-Pressigny.

Les décomptes technologiques des différents niveaux suffisent à mettre en évidence l'un des deux traits marquants des séries lithiques qui est le fractionnement des chaînes opératoires. En amont, la sous-représentation des produits corticaux montre que les blocs sont parvenus dans le gisement principalement sous la forme de modules déjà par- tiellement décortiqués. Cela est particulièrement marqué pour le silex blond de la craie. En effet, dans ce matériau, les éclats de décorticage sont rares et représentés presque exclusivement par des pièces où le cortex n'offre qu'une plage très réduite. Les lames à crête sont également presque absentes. Dans certains cas (silex tertiaire allochtone et certaines catégories de silex marins d'origine non déterminée), le silex est arrivé à l'état de support déjà débité, voire même d'outils déjà façonnés. Cela est caractéristique de chaînes opératoires des gisements où la matière première était importée, à grande distance (comme par exemple sur la plupart des gisements du Paléolithique supérieur d'Auvergne ; Bracco 1992 et 1996 ; Surmely 1998). Ce phénomène est à rapprocher de la circulation de nucléus prêts à débiter durant le Magdalénien (Morala 1999 ; Surmely et al. 2002). Pour le silex pressignien, on observe toutefois quelques cas d'importation de blocs bruts qui ont été exploités sur place (fig. 9). Les deux remontages principaux, qui intègrent respectivement 63 et 31 pièces, concernent ce matériau (fig. 9 à 11).

Le second caractère marquant des chaînes opératoires lithiques du Protomagdalénien du Blot est la gestion rigoureuse de la matière première, qui s'explique aussi par l'éloignement des sources d'approvisionnement. On constate en effet que les outils ont été ravivés, souvent plusieurs fois (fig. 12 et 13). Nous reviendrons par la suite, dans le paragraphe consacré à la typologie, sur l'incidence de ces transformations. Dans le même ordre d'idée, il faut signaler l'emport, hors du gisement, des éléments récupérables, et notamment des nucléus. Les niveaux protomagdaléniens n'ont en effet livré que deux petits nucléus en silex de la craie, dont un réutilisé en petit abraseur. On pourrait bien évidemment objecter que ces pièces ont pu être plus simplement rejetées hors de la partie centrale de l'abri, mais cette hypothèse est contredite par le fait que cette absence de nucléus est commune à l'ensemble des gisements du Paléolithique supérieur de la moyenne montagne auvergnate que nous avons pu étudier (Surmely 1998 ; Surmely 2000). A l'évidence, les hommes, lors de 

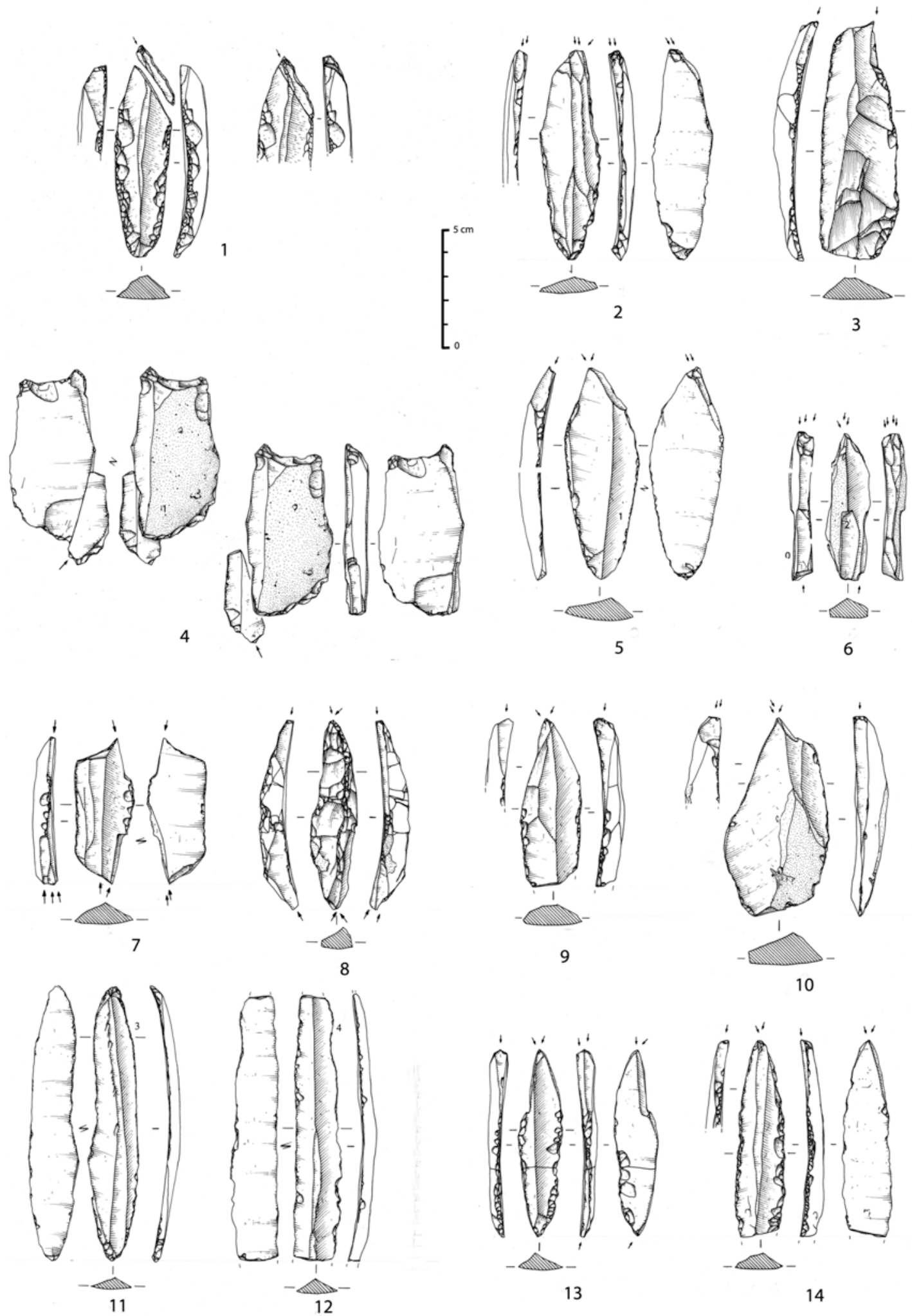

Figure 12 - Le Blot - Protomagdalénien. Outils de fonds commun. Dessins Ph. Alix.

Figure 12 - Le Blot -Protomagdalenian. Commonly found tools. Drawings Ph. Alix. 

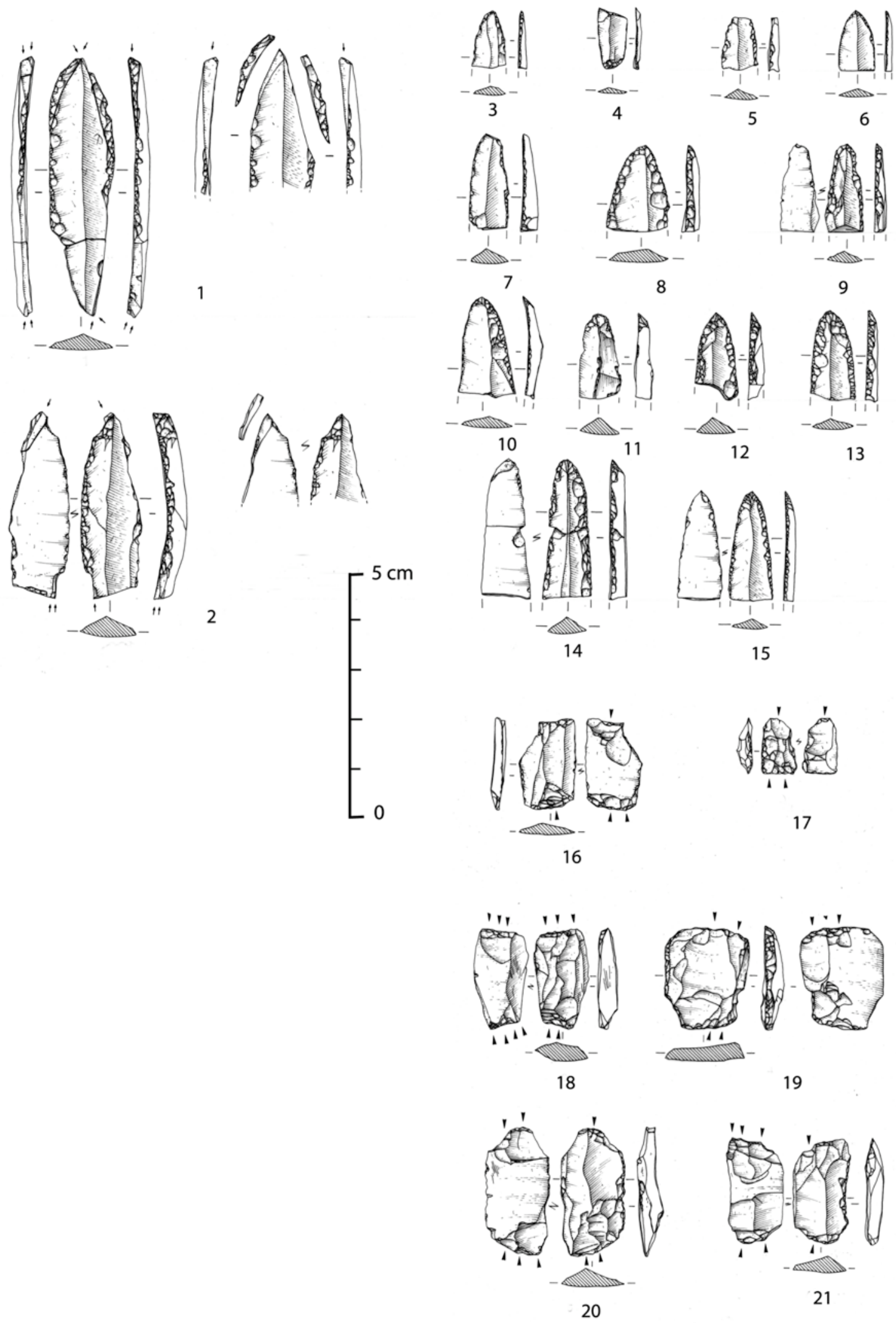

Figure 13 - Le Blot - Protomagdalénien. Outils de fonds commun. Dessins Ph. Alix.

Figure 13 - Le Blot - Protomagdalénien. Outils de fonds commun. Dessins Ph. Alix. 
leur départ du gisement, ont emporté avec eux les produits encore utilisables, parmi lesquels figuraient les nucléus. Pour nous, ce comportement est à mettre en relation avec la mobilité des groupes humains et une gestion rigoureuse de matières premières d'origine éloignée.

Cette récupération concerne également d'autres pièces, comme des outils et des lames brutes. II existe en effet un déficit sensible de produits laminaires, par rapport à ce qui a pu être débité sur place. Pourtant, et c'est là où le Blot se distingue nettement des autres gisements de la moyenne montagne auvergnate et notamment du gisement voisin de Béraud (attribué à l'Epipaléolithique ancien, Surmely et al. sous presse), ce souci de récupération n'est pas systématique. Les fouilles ont permis en effet de mettre au jour des pièces entières, qui paraissent encore tout à fait utilisables ou transformables. L'exemple le plus frappant est la lame appointée de $18 \mathrm{~cm}$ de long, réalisée dans du silex pressignien (fig. 8-3). Considérant les comportements évoqués plus haut et le haut degré de ravivage de la majorité des outils, on peut s'étonner de l'abandon sur place d'une telle pièce. Plusieurs réponses peuvent être apportées à cette interrogation, parmi lesquelles l'hypothèse de comportements «cultuels », échappant à la simple logique rationaliste ou bien celle d'une réserve pour un séjour ultérieur qui n'eût jamais lieu.

\section{Objectifs et conduite de la production lithique}

\section{La production laminaire}

La production lithique a été incontestablement tournée vers la réalisation de grandes lames, souvent assez robustes et peu arquées, même si la fragmentation de celles-ci et leur transformation en outils nous empêchent de donner des chiffres précis pour ce qui est de la longueur. II s'agit là d'un trait marquant du Protomagdalénien (Peyrony 1938), et qui se retrouve de façon très nette dans les autres gisements, tels que Pataud ou Laugerie-Haute (Bosselin 1992 et 1997 ; Bordes 1978 ; Clay 1995 ; Delporte 1972 ; Demars 1994 ; Kong-Cho 1997 ; Peyrony 1938). Cette culture de la grande lame distingue nettement le Protomagdalénien des faciès culturels qui l'ont immédiatement précédé ou suivi. C'est le cas notamment au Blot et à Laugerie-Haute-Est (Demars 1994). On peut penser que c'est cette finalité de débitage de grande taille qui a induit le recours à des matières premières allochtones propices à cet usage.

En l'absence de remontages significatifs et de nucléus, il est difficile de reconstituer précisément les processus opératoires. Ceux-ci semblent être voisins des autres faciès culturels à grandes lames, comme le magdalénien évolué, avec Étiolles et les Tarterêts comme références principales. L'examen des négatifs d'enlèvement sur les lames montre nettement que le débitage unipolaire a été privilégié, le bipolaire étant apparemment réduit le plus souvent à une fonction marginale de rectification de la convexité longitudinale de la table laminaire.

L'une des caractéristiques du Protomagdalénien du Blot est la fréquence de l'aménagement d'éperons pour le détachement des lames (fig. 12-2 et 12-11). Ce processus a été analysé en détail dans un autre article (Surmely et Alix 2005) et nous le décrirons ici très brièvement. Les Protomagdaléniens ont eu largement recours à l'aménagement en éperon des talons. Ces éperons, souvent assez discrets, sont parfois massifs et proéminents (atteignant une hauteur de $5 \mathrm{~mm}$, pour une largeur de $10 \mathrm{~mm}$ ). conjuguée à la proéminence et à la position très en avant de l'éperon, a entraîné un véritable arrachement de la lame en arrière du point d'impact, donnant à la partie proximale de la lame une morphologie caractéristique, avec un talon éversé, une lèvre très marquée et une absence de bulbe de percussion (qui est parfois même concave).

Les talons en éperon se retrouvent exclusivement sur les lames et surtout sur les beaux produits de plein débitage, ce qui explique qu'ils sont surtout associés aux outils. Ils se retrouvent aussi sur des lames à crête ou d'entame. Ils sont totalement absents sur les éclats. La fonction des éperons correspond à la volonté de renforcer le plan de frappe, pour réduire sensiblement le risque d'éclatement ou d'esquillement de celui-ci et faciliter ainsi le débitage de belles lames planes.

Il est difficile de connaître leur fréquence exacte, compte tenu de leur élimination postérieure dans de nombreux cas. Un décompte permet toutefois d'observer qu'ils constituent $51 \%$ des talons conservés des lames non corticales, pour l'ensemble des niveaux protomagdaléniens. Ce pourcentage est de $20 \%$ pour le niveau 36 et de $46 \%$ pour le petit niveau 38 de Laugerie. Ce pourcentage est assurément inférieur à la réalité initiale, compte tenu de leur élimination préférentielle avant utilisation du support. Les éperons concernent tous les types de silex.

Les préhistoriques ont cherché le plus souvent à faire disparaître la saillie gênante (notamment pour l'emmanchement) que formait le talon en éperon, notamment quand celui-ci était très massif. Le moyen le plus fréquemment utilisé était celui de l'aménagement d'une lame appointée (aussi appelée grattoir ogival, selon les auteurs), ellemême servant de base pour la fabrication d'un burin. Cette élimination quasi systématique des talons explique qu'ils soient rarement conservés.

Dans le même temps, il est à noter que l'aménagement d'éperons a eu pour effet, surtout dans le cas des types massifs, la création d'un rentrant important dans le plan de frappe, ce qui a nécessité de fréquents ravivages, sous la forme de tablettes ou d'éclats épais et donc une consommation plus importante de matière première (Pèlegrin 1992 ; Pigeot 2004).

Dans le cas du Blot, on observe que les éperons ont été aménagés à partir d'un plan de frappe très oblique qui tend à se rapprocher de l'horizontale au fur et à mesure des ravivages par le biais de tablettes et surtout d'éclats qui deviennent de plus en plus petits et ont parfois l'aspect de petites pièces esquillées et sont tirés de l'avant du nucléus. Dans certains cas, le tailleur a été amené à surcreuser le plan de frappe pour lui donner une obliquité partielle, par le biais de tous petits éclats. Le remontage prin- 
cipal (fig. 10 et 11) montre que la situation a été rétablie, au prix d'une diminution sensible de la longueur des lames obtenues, par l'enlèvement de tablettes et éclats épais, extraites à partir d'un des flancs. Cette séquence a même été répétée deux fois, ce qui témoigne du souci d'exploitation rigoureuse du nucléus.

\section{Morphologie des lames}

La très forte fragmentation des produits laminaires, due au bris lors du détachement et surtout de la transformation en outils, empêche de dresser un inventaire précis des longueurs de lames. Les critères statistiquement utilisables sont seulement ceux de la largeur et de l'épaisseur. On observe que les modules de lames réalisées dans le silex pressignien sont en moyenne plus larges que ceux des produits façonnés dans le silex de la craie.

\section{La production lamellaire}

La production de lamelles reste une question non résolue. Les lamelles brutes sont rares. Les supports des très nombreuses lamelles à dos paraissent avoir été des produits assez courts, assez peu épais et larges, parfois irréguliers, et plus proches des esquilles lamellaires que des lamelles véritables. Les lamelles des autres gisements protomagdaléniens que nous avons observées présentent les mêmes caractéristiques et procèdent vraisemblablement du même processus technique, même si les études antérieures (Bricker 1995 ; Bosselin 1992 et 1997 ; Kong-Cho 1997) n'ont guère abordé cet aspect. Le caractère particulier des séries lithiques du Blot, et notamment la disparition des nucléus, nous empêche de déterminer avec précision les modalités de production de lamelles à partir de nucléus véritables et l'existence d'une continuité éventuelle entre production laminaire et production lamellaire. A notre avis, cette question devra d'abord être éclaircie sur les gisements où les nucléus sont présents, avant d'être étudiée au Blot.

La morphologie de certains supports transformés en armatures laisse penser que des chutes de burin ont pu être utilisées à cette fin, par sélection des exemplaires les plus aptes à remplir cette fonction. Mais, il n'existe pas, à notre avis, d'exploitation spécialisée à cette fin de « burinsnucléus ", du type de ceux individualisés dans le Gravettien sous-jacent (Klaric 1999). De même, il n'existe aucune pièce du type " burin du Raysse » (Klaric et al. 2002) et les lamelles ne présentent pas de vestiges de la " retouche tertiaire du biseau ».

En effet, la morphologie des burins du Blot montre que l'existence de plusieurs enlèvements sur de nombreuses pièces s'explique avant tout par une volonté de ravivage des outils, ce qui est confirmé par les analyses tracéologiques réalisées par $\mathrm{M}$. Hays.

II semblerait donc plus juste, en première analyse, de parler de récupération opportuniste de chutes de burins, que d'évoquer une éventuelle production spécialisée de lamelles à partir de supports laminaires épais.

\section{Observations typologiques}

Une caractérisation typologique détaillée a été faite par $B$. Bosselin (1992). Nous donnons ici le résultat de nos observations personnelles.

\section{Observations générales}

La première observation porte sur le type de supports utilisés pour la transformation en outils. D'une façon générale, les outils de fonds commun ont été aménagés de façon quasi exclusive sur des lames. Les déchets de débitage, tablettes, outils, ont été dédaignés, à l'exception de quelques rares pièces retouchées ou utilisées comme pièces esquillées.

En second lieu, il convient de rappeler une fois de plus, en préalable aux observations qui suivent, le très fort degré de ravivage et de fragmentation des outils. Cela constitue bien évidemment un frein sérieux à l'étude typologique classique, mais permet également d'éclairer certains processus évolutifs.

Le « remontage » de ravivages d'outils montre en effet la succession de plusieurs types d'outils. La séquence la plus fréquente, bien représentée sur le gisement du Blot, est celle qui conduit de la lame appointée au burin dièdre sur cassure via le burin sur troncature et le burin dièdre d'axe (cf. infra).

Ce comportement, déjà remarqué sur plusieurs gisements et noté par D. Buisson à propos des niveaux gravettiens (Buisson 1991), permet de limiter la portée informative et surtout comparative des listes typologiques classiques, dont la composition nous paraît dépendre autant du degré de ravivage des outils (donc du degré des supports et par là de l'éloignement plus ou moins grand des sources de matières premières) que du faciès culturel proprement dit.

En allant plus loin dans cette réflexion, il est même possible de subodorer une fonction technique à certaines pièces présentées comme des outils.

La retouche protomagdalénienne

II s'agit d'une retouche écailleuse, proche de celle rencontrée dans le moustérien. Ce type de retouche, qui est pratiquement inconnu dans le gravettien, a constitué un des éléments distinctifs de la culture protomagdalénienne. Il convient de signaler également que la retouche protomagdalénienne a été appliquée le plus souvent aux deux bords de la pièce ( $71 \%$ des pièces), alors que la proportion est presque exactement inverse dans le cas des autres types de retouches (retouche sur un seul bord sur $74 \%$ des pièces) (fig. 13). A l'évidence, la retouche dite protomagdalénienne joue un rôle distinct des autres retouches courantes.

Nous avions d'abord pensé à une retouche à but technologique, destinée à régulariser le bord des lames, dans le but de faciliter l'enlèvement des chutes de burins. Ce raisonnement se basait sur le fait que les burins étaient majoritairement aménagés sur des lames à retouches protomagdaléniennes, observation vérifiée également pour les 
séries de l'Abri Pataud. Mais C. Sestier (com. orale) nous a démontré qu'une retouche simple aurait été suffisante pour cet objectif. La retouche écailleuse, complexe, correspond plus probablement à une volonté de ravivage du (ou des) tranchant(s) de la pièce. II est donc possible de mettre en relation la mise en œuvre de ce type de retouches avec la gestion rigoureuse de la matière première et de la production laminaire. Ainsi s'expliquerait également le fait que la retouche protomagdalénienne ait été appliquée majoritairement sur les deux bords de la pièce, dans un but d'exploitation maximale.

On pourrait objecter que ce type de retouches se rencontre également dans des sites du Périgord où la matière première était de provenance beaucoup moins lointaine (silex du Bergeracois), voire sub-locale (silex sénonien blond ou noir de Dordogne). Mais, même dans les cas où le silex était d'origine proche, il n'en demeurait pas moins que la production de supports laminaires de grande taille, avec l'aménagement de talons en éperon, restait un objectif difficile à atteindre et assez lourd en terme de mise en œuvre. Les produits obtenus devaient donc rester assez précieux, ce qui pourrait expliquer qu'ils ont été ravivés beaucoup plus fortement que ceux, moins imposants, que d'autres faciès culturels. II faut ajouter que la mise en œuvre de la retouche écailleuse, au-delà de son utilité fonctionnelle pour certains types de matières premières, a pu être généralisée, en devenant un comportement habituel.

\section{Les burins}

Les burins sont les outils les mieux représentés sur le site du Blot, comme sur les autres gisements protomagdaléniens (Bosselin 1992) avec 36 à $52 \%$ de l'ensemble des outils de fonds commun (fig. 16). Ces pièces sont presque exclusivement aménagées sur des lames. Les quelques exemplaires qui sont parvenus entiers jusqu'à nous montrent la recherche de supports de belles dimensions, très probablement en vue de l'emmanchement.

Comme nous l'avons fait observer plus haut, ces classements typologiques classiques, établissant une distinction minutieuse des différents types, nous semblent ici peu pertinents. En effet, les remontages montrent que la forme du burin correspond en fait, avant tout, à sa position dans un cycle de transformation progressive du support initial. Du burin sur troncature, la pièce devient un burin dièdre par enlèvement d'une ou plusieurs chutes, avant de terminer, souvent, à l'état de burins doubles ou de burins sur cassure, dans le cas de pièces brisées lors de l'utilisation. Au Blot, pour des raisons tenant à l'origine lointaine des matières premières, le degré d'exhaustion des outils a été particulièrement fort, ce qui se traduit logiquement par une plus forte proportion de burins dièdres, nucléiformes, doubles, mixtes... que dans les autres gisements du Protomagdalénien du Périgord où une partie au moins de la matière première était d'origine locale ou sublocale. Sans remettre en question de façon générale la classification habituelle des divers types de burins, il nous semble que dans le cas du gisement du Blot, ces distinctions semblent peu informatives et en tout cas ne peuvent constituer un instrument comparatif à l'état brut.
L'étude tracéologique montre que le taux d'utilisation des burins est de $71 \%$, ce qui est assez important.

Les lames appointées (fig. 13-3 à 13-15)

Les lames appointées sont formées de supports présentant une pointe régulière mais jamais très aiguë, dégagée par des retouches le plus souvent écailleuses qui se prolongent parfois sans rupture sur les bords de la lame. Certains auteurs (Bosselin 1992) n'ont pas voulu distinguer ces pièces du groupe plus large des lames retouchées, ce qui nous semble une erreur, compte tenu du caractère volontaire et soigné de la retouche apicale. D'autres les ont regroupées dans la catégorie des grattoirs, en y ajoutant le qualificatif " ogival ". D'autres encore parlent de « pointes ». A la suite d'H. Delporte et de F. Bordes, nous pensons que les lames appointées, de par leurs caractères spécifiques, constituent un groupe distinct des lames retouchées, des grattoirs et des véritables pointes.

L'abondance des lames appointées, quelles que soient leurs dénominations, constitue un trait caractéristique du Protomagdalénien (6 à $13 \%$ des outils de fonds commun, selon les « phases » d'occupation). Au Blot, l'aménagement de la pointe a été réalisé, dans plus de $70 \%$ des cas, sur l'extrémité proximale de la lame. Le plus bel exemple est celui de la lame appointée, aménagée sur la plus grande lame entière de $18 \mathrm{~cm}$ retrouvée sur le site (fig. 8-3). Ce caractère se retrouve à l'identique sur les autres sites étudiés, tels que LaugerieHaute-Est. II ne s'agit pas donc d'un hasard et l'on peut supposer, comme nous l'avons déjà dit plus haut, que la mise en forme de la pointe a permis d'éliminer la saillie très marquée que constituaient les talons en éperon et qui devait représenter un obstacle ou tout du moins une gêne importante pour l'emmanchement des lames.

La deuxième information est apportée par l'étude tracéologique qui montre que les lames appointées retrouvées sur le site ne portent pas de traces fonctionnelles et semblent n'avoir pas servi (ou pas encore servi). Ainsi, la proportion de pièces portant des traces d'utilisation est seulement de 37 $\%$. Ce chiffre est à comparer avec les $71 \%$ de pièces utilisées pour les burins et même $82 \%$ pour les pièces esquillées. Ajoutons que les utilisations présumées sont variables, ce qui indique qu'il n'y avait pas de fonction définie pour ces pièces, tout en montrant un spectre d'utilisation globalement assez proche de celui des burins.

Enfin, on observe, dans de très nombreux cas, que la lame appointée est transformée en burin, ce qui est parfaitement observable quand il est possible de faire le remontage des chutes (fig. 12-1, 12-13 et 13-1).

De ces observations, il est possible de douter que les lames appointées aient un rôle fonctionnel réel ou spécifique. II faut donc envisager l'hypothèse que l'aménagement de ces pièces ait eu surtout une fonction de façonnage de la lame, permettant d'éliminer la saillie du talon et de régulariser l'extrémité afin de permettre l'obtention d'un burin régulier, ce qui était encore amélioré par la présence d'une retouche latérale. Les lames appointées pourraient donc être avant tout des « pièces techniques ". 
(chiffre en haut : NB ; chiffre en bas : Nc)

\begin{tabular}{|l|c|c|c|c|c|c|c|c|}
\hline $\begin{array}{l}\text { largeur } \\
\text { epaisseur }\end{array}$ & $\mathbf{0 - 1}$ & $\mathbf{1 - 2}$ & $\mathbf{2 - 3}$ & $\mathbf{3 - 4}$ & $\mathbf{4 - 5}$ & $\mathbf{5 - 6}$ & Total & $\begin{array}{c}\text { Total en } \\
\%\end{array}$ \\
\hline $\mathbf{0 - 1}$ & & 1 & & & & & $\mathbf{1}$ & $\mathbf{1}$ \\
\hline $\mathbf{1 - 2}$ & & 20 & 39 & 7 & 3 & & $\mathbf{6 9}$ & $\mathbf{8 1}$ \\
& & 9 & 36 & 12 & 3 & 1 & $\mathbf{6 1}$ & $\mathbf{7 7}$ \\
\hline $\mathbf{2 - 3}$ & & & 5 & 8 & 1 & 1 & $\mathbf{1 5}$ & $\mathbf{1 8}$ \\
& & & 6 & 6 & 4 & 1 & 17 & $\mathbf{2 1}$ \\
\hline $\mathbf{3 - 4}$ & & & & & & & & \\
\hline $\mathbf{4 - 5}$ & & & & & & & & \\
& & & & & & & & \\
\hline Total & & $\mathbf{2 1}$ & $\mathbf{4 4}$ & $\mathbf{1 5}$ & $\mathbf{4}$ & $\mathbf{1}$ & $\mathbf{8 5}$ & $\mathbf{1 0 0}$ \\
& & $\mathbf{9}$ & $\mathbf{4 3}$ & $\mathbf{1 8}$ & $\mathbf{7}$ & $\mathbf{2}$ & $\mathbf{7 9}$ & $\mathbf{1 0 0}$ \\
\hline Total en & & $\mathbf{2 5}$ & $\mathbf{5 2}$ & $\mathbf{1 8}$ & $\mathbf{5}$ & $\mathbf{1}$ & $\mathbf{1 0 0}$ & \\
\% & & $\mathbf{1 1}$ & $\mathbf{5 4}$ & $\mathbf{2 3}$ & $\mathbf{9}$ & $\mathbf{3}$ & $\mathbf{1 0 0}$ & \\
\hline
\end{tabular}

Figure 14 - Le Blot - Protomagdalénien. Dimensions des microlithes (échantillon).

Figure 14 - Le Blot -Protomagdalenian. Microlith dimensions (sample).

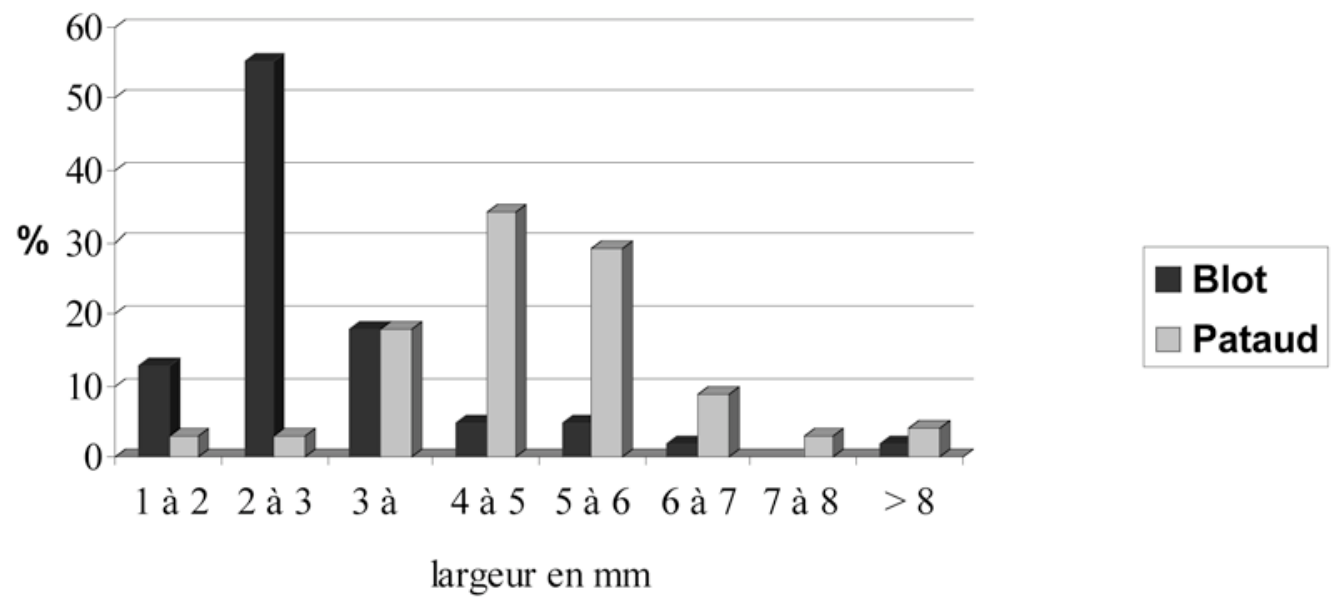

Figure 15 - Comparaison des largeurs des lamelles à dos entre les niveaux protomagdaléniens de l'abri Pataud et du Blot (en $\%$ pour chaque site).

Figure 15 - Comparison of the widths of backed blades between the protomagdalenian levels at l'abri Pataud and le Blot (\% for each site).

\section{Les grattoirs}

Les véritables grattoirs, que nous distinguons des lames appointées (cf supra), sont très rares sur le gisement du Blot, comme dans l'ensemble des séries protomagdaléniennes (Bosselin 1992). Ceci pose bien évidemment le problème du recours éventuel à d'autres types d'outils pour les travaux de préparation des peaux.
Les lames retouchées et encochées

De nombreux supports comportent des esquillements sur les bords, qui sont souvent discontinus. Comme toujours, ceci pose le problème de la détermination de l'origine de ces traces. S'agit-il de stigmates post-dépositionnels (ce qui semble être dans la majorité des cas), de traces d'utilisation (= supports bruts utilisés, selon la terminologie typologique 


\begin{tabular}{|c|c|c|c|c|c|c|c|}
\hline Phase & Nombre & $\begin{array}{c}\text { Burins } \\
(\mathbf{\%})\end{array}$ & $\begin{array}{c}\text { Lames } \\
\text { appointées } \\
(\%)\end{array}$ & $\begin{array}{c}\text { Grattoirs } \\
(\mathbf{\%})\end{array}$ & $\begin{array}{c}\text { Pièces } \\
\text { esquillées } \\
\mathbf{( \% )}\end{array}$ & $\begin{array}{c}\text { Perçoirs } \\
\text { et bec } \\
\mathbf{( \% )}\end{array}$ & $\begin{array}{c}\text { Lames } \\
\text { retouchées } \\
\text { et } \\
\text { encochées } \\
(\%)\end{array}$ \\
\hline $\mathbf{2}$ inf & 64 & 36 & 6 & 0 & 19 & 4,5 & 23,5 \\
\hline $\mathbf{2}$ sup & 130 & 41,5 & 11,5 & 1,5 & 6 & 4 & 33 \\
\hline $\mathbf{3}$ & 54 & 52 & 13 & 0 & 7,5 & 0 & 18,5 \\
\hline
\end{tabular}

Figure 16 - Le Blot - Protomagdalénien. Répartition des outils de fonds commun par «phases » d'occupation.

Figure 16 - Le Blot -Protomagdalenian. Distribution of common tools by occupational «phases ».

traditionnelle), ou bien de retouches (= outils, selon la détermination traditionnelle) ? Seule la tracéologie peut permettre, au cas par cas, de répondre à cette interrogation.

Les lames retouchées indubitables et encochées sont nombreuses. Elles comportent une retouche qui se partage, en proportions presque égales, entre type écailleux (= retouche protomagdalénienne) et types standards. Comme nous l'avons déjà mentionné, les retouches écailleuses concernent majoritairement les deux tranchants des pièces, alors que les retouches d'autres types n'affectent généralement qu'un seul bord.

Les pièces esquillées (fig. 13-18 à 13-21)

L'importance numérique des pièces esquillées est un autre trait caractéristique des séries protomagdaléniennes du Blot, comme l'avaient déjà noté les chercheurs précédents (Bosselin 1992.

Elles représentent 6 à $19 \%$ des outils de fonds commun. L'étude tracéologique a confirmé leur rôle fonctionnel. Elle montre aussi que le taux de pièces présentant des traces d'utilisation est très élevé (82 \%), ce qui tient peut-être à des raisons taphonomiques (les usages de la percussion sont sans doute ceux qui sont les plus apparents), mais aussi indiscutablement fonctionnelles. Elles sont aménagées presque exclusivement sur des tronçons de lames, qui correspondent certainement à des pièces mises au rebut.

\section{Les armatures microlithiques}

Les armatures microlithiques présentent deux caractéristiques essentielles qui ont été remarquées par l'ensemble des chercheurs ayant étudié les séries :
- leur importance numérique. Les armatures microlithiques sont, en nombre, les outils les plus abondants dans l'ensemble des niveaux protomagdaléniens du Blot (55 à $70 \%$ de l'ensemble des outils, selon Bosselin 1992). Plusieurs auteurs ont souligné cette abondance en la rapprochant de celle observée dans les niveaux gravettiens sous-jacents et en l'opposant aux taux beaucoup plus faibles de microlithes des gisements périgourdins. Mais cette représentation exceptionnelle s'explique d'abord indiscutablement par le fait qu'un tamisage à l'eau systématique a permis, au Blot, de recueillir la quasi intégralité des exemplaires, y compris des fragments véritablement minuscules, alors que les fouilles des gisements périgourdins, y compris à l'Abri Pataud ${ }^{9}$, se sont faites sans cette opération. Les travaux de L. Chiotti et R. Nespoulet à l'Abri Pataud (Nespoulet et al. 2008), ou de J.-G. Bordes à l'Abri Caminade opération (Bordes et Lenoble 2002) ont permis de comparer les résultats dans ce domaine entre des méthodes récentes avec tamisage et des travaux des années 50 sans cette opération ;

- le second critère distinctif est celui de la taille (fig. 14 et 15). Les lamelles à dos du Blot se caractérisent en effet par des dimensions remarquablement réduites. $68 \%$ des pièces ont une largeur inférieure à $3 \mathrm{~mm}$ et $86 \%$ une épaisseur inférieure à $4 \mathrm{~mm}$ (fig. 14). Ceci fait des armatures du Blot des pièces nettement plus petites que celles trouvées dans bien d'autres gisements (Bosselin 1992), bien que les différences dans la collecte amenuisent singulièrement la portée de cette comparaison (note 10). Dans le Protomagdalénien de l'Abri Pataud (fig. 15), les valeurs respectives sont 2,5 et 47 $\%$, proches de celles relevées sur le gisement du magdalénien final du Pont-de-Longues (3\% ont une largeur inférieure à $3 \mathrm{~mm}$; Surmely et al. 2002). Ce pourcentage n'atteint pas $10 \%$ sur le gisement magdalénien moyen de Thônes

(9) Les conditions de collecte (présence ou absence d'un tamisage, tamisage à l'eau ou non, taille de la maille du tamis) ont une incidence certaine sur le nombre, mais aussi sur la taille des microlithes découverts. Cela amène à relativiser la portée des comparaisons morphométriques des armatures entre des gisements fouillés de façon différente, notamment sur les toutes petites pièces. A l'Abri Pataud, les fouilles anciennes du niveau 2 ont été faites sans tamisage pour la campagne 1958 (trenches I à VI) et avec tamisage à sec pour la campagne 1963 (trenches VII) (com. orale L. Chiotti). Les recherches récentes conduites par L. Chiotti et $R$. Nespoulet dans le même horizon, accompagnées d'un tamisage à l'eau, ont mis au jour une industrie dans laquelle les armatures microlithiques représentent plus de $90 \%$ de l'outillage (Nespoulet et al. 2008). 
(Grandeyrolles, Puy-de-Dôme ; Surmely 1998). Les pièces des gisements magdaléniens du Bassin Parisien, malgré des différences entre sites, sont également en moyenne bien plus larges que les armatures protomagdaléniennes du Blot (Valentin 1995 ; Debout 2003).

Cette petite taille pourrait s'expliquer en premier lieu par la technique de fabrication du support, qui semble faire largement appel à des chutes de burin, détachées de burins véritables et d'éclats épais. Ce point reste toutefois à vérifier.

La troisième caractéristique est typologique. Selon B. Bosselin, les armatures du protomagdalénien ne sont pas des microgravettes mais plutôt des lamelles à dos. Mais cette qualification repose sur la définition typologique restrictive adoptée par ce chercheur ${ }^{10}$.

Avec nos critères (cf. supra), qui placent les fragments mésiaux dans la catégorie des armatures de type indéterminé, nous trouvons $12 \%$ de microgravettes, $7 \%$ de pointes à dos simple, $5 \%$ de lamelles à dos, et $75 \%$ d'armatures de type indéterminé (essentiellement des fragments mésiaux). Notons enfin que le caractère " pointu » est parfois difficile à apprécier sur les plus petites armatures.

Ces caractéristiques, tant quantitatives que morphologiques amènent évidemment à poser la question de l'usage de ces armatures microlithiques. Leur importance et leur standardisation tendent à indiquer qu'il s'agissait d'outils dont la fonction était à la fois bien précise et importante dans la vie quotidienne des populations. Leurs petites dimensions ne plaident pas en faveur d'une utilisation comme armatures de couteaux. En l'absence d'étude tracéologique sur ces pièces, il est difficile d'en dire plus. $\mathrm{Si}$ les plus grandes peuvent correspondre à des armatures de projectile, placées en tête ou en barbelure latérale d'une hampe dont le mode de propulsion reste impossible à préciser, la question se pose de l'usage des plus petites, certaines étant si minuscules qu'elles sont difficiles à saisir.

\section{Protomagdalénien ou Périgordien VII}

Sur tous les plans, le Protomagdalénien du Blot présente beaucoup d'analogies avec les quelques autres gisements de cette époque recensés à ce jour : Abri Pataud, LaugerieHaute-Est (Dordogne), Les Peyrugues (Lot). Les différences observées, comme les proportions de microlithes, tiennent assurément à la méthodologie retenue pour la fouille et notamment la mise en œuvre ou non d'un tamisage à l'eau.

Reste la question, déjà abordée par de nombreux auteurs (notamment Bosselin 1992), des liens éventuels qui ont pu exister entre les différents gisements. Si l'existence de liens directs entre l'Abri Pataud et Laugerie-Haute, voire les Peyrugues, peut être tenue pour possible, voire très probable (note 5), il n'en est pas de même pour Le Blot. Aucun indice matériel ne permet en effet de supposer l'existence d'un lien direct ou d'une communication quelconque entre le Blot et les autres gisements cités. La dis- tance qui les sépare est de plus de $300 \mathrm{~km}$ à vol d'oiseau avec, en outre, l'obstacle supplémentaire des monts d'Auvergne. Aucune pièce lithique retrouvée au Blot ne peut provenir d'Aquitaine ou du Quercy. Toute la matière première utilisée au Blot provient de Touraine.

Les parentés étroites observées entre les différentes industries lithiques semblent donc s'expliquer par des convergences culturelles, sans relation physique entre les deux groupes de gisements. Le seul lien possible réside dans la présence très probable, en très faibles quantités, de silex pressigniens dans les séries de l'Abri Pataud (obs. personnelles), ce qui pourrait accréditer l'idée d'un berceau ou d'un lieu de rassemblement ou d'échanges au cours du Protomagdalénien dans ce secteur. Cette hypothèse reste toutefois très fragile.

Cette unité du Protomagdalénien contraste aussi avec les cultures antérieures.

La plupart des chercheurs ont minimisé le caractère spécifique du Protomagdalénien, individualisé par D. Peyrony, pour en faire un simple faciès évolutif du Gravettien final (Périgordien VII). Leurs arguments portent principalement sur la présence de quelques pointes de La Gravette dans les niveaux protomagdaléniens et l'abondance, dans les deux faciès, des microlithes.

Cette argumentation nous semble à revoir. En effet, la complexité stratigraphique des différents gisements, et avant tout celui du Blot, a pu faciliter la migration de quelques pièces isolées d'un niveau à l'autre. Mais surtout, le Protomagdalénien s'individualise nettement des cultures antérieures, au Blot comme ailleurs, par de très nombreux critères : débitage laminaire de grande taille avec aménagement fréquent en éperon des talons, importance de la proportion en matières premières exogènes, retouche spécifique... Du point de vue typologique, le Protomagdalénien se distingue également par une composition originale d'outils : abondance des burins, des lames retouchées et des pièces esquillées, présence de lames appointées. Enfin, la forte proportion de microlithes dans les deux cultures ne saurait être considérée comme un argument fiable de continuité. Selon B. Bosselin (1992), qui a étudié les armatures des deux faciès, ces dernières seraient différentes.

Tout ceci signe, selon nous, l'individualité manifeste du Protomagdalénien comme faciès culturel, distinct du Gravettien récent et, dans une moindre mesure, du Solutréen, même si la méconnaissance du Solutréen inférieur nous prive de possibilités de comparaison précises. Comme le note B. Bosselin, qui parle de " coupure typologique " (Bosselin 1992), le lien phylétique entre le Gravettien et le Protomagdalénien du Blot est impossible à établir et la redénomination du Protomagdalénien comme "Gravettien final », suggérée par certains chercheurs (Djinjian et al. 1999), nous paraît très discutable. Les différences évoquées plus haut sont en effet bien plus importantes que les quelques points de convergence (rareté des grattoirs, abondance des armatures micro-

(10) La matière première utilisée aux Peyrugues comprend une proportion importante de silex du Bergeracois (com. orale M. Allard). 
lithiques). La culture la plus proche du Protomagdalénien nous paraît être le Magdalénien supérieur, en raison de nombreux critères de convergence (production laminaire soignée, talons en éperon massifs, abondance des armatures microlithiques et des burins), même si une continuité véritable est impossible, compte tenu des millénaires qui séparent ces deux cultures. En tenant compte de cette dernière observation, le terme de Protomagdalénien nous paraît donc tout à fait justifié pour désigner ce faciès culturel.

Enfin, aucune différence sensible ne peut être observée, du point de vue de l'industrie lithique, entre les différents niveaux protomagdaléniens du Blot et notamment entre les trois phases distinguées lors de la fouille. C'est également le constat fait par l'ensemble des chercheurs ayant étudié les séries (Bosselin 1992 ; Chauvière et Fontana Sous presse 1). Ceci, appuyé par l'existence de quelques remontages entre pièces de niveau différents, la réutilisation de la "structure » de blocs d'une phase à l'autre, plaiderait en faveur d'une faible dispersion dans le temps des différents niveaux d'occupation, sous forme de séjours saisonniers répétés et assez courts, à moins que ce caractère ne soit imputable à un remaniement des niveaux non décelé lors de la fouille.

\section{CONCLUSION}

Le Gravettien est peu représenté en Auvergne. Toutefois, la présence de faciès assez rares (Gravettien ancien, Protomagdalénien) et l'importance du gisement de plein air du Sire, laissent penser que ce fait est imputable avant tout au faible développement de la recherche et à de mauvaises conditions taphonomiques, plus qu'à une faible attractivité de la région à l'époque considérée. Les dates obtenues au Sire placent le site parmi les plus anciennes manifestations du Gravettien en Europe, même si l'incertitude des calibrations des dates ${ }^{14} \mathrm{C}$ incite à beaucoup de prudence dans les comparaisons.

Toujours à propos du même site, le poids écrasant du Cheval dans la faune chassée et les modalités de gestion de ce gibier, évoquent les occupations de La Vigne-Brun (Loire) et de Solutré (Saône-et-Loire) tout en réaffirmant l'importance cynégétique du Cheval dans la France du centre-est et la place secondaire qu'occupait le Renne, ce qui jette le doute sur la prétendue migration estivale des rennes et son importance économique en Auvergne durant cette période.

Il convient ensuite de revenir sur le Protomagdalénien, culture rarement identifiée et qui est pourtant bien présente en Auvergne. Comme nous l'avons vu, ce faciès culturel présente une grande originalité, qui le démarque du Gravettien. Malgré de très fortes ressemblances, aucun indice matériel ne permet d'aller dans le sens de contacts physiques entre les occupants du gisement du Blot et ceux des autres sites contemporains du Bassin aquitain. La genèse et le développement du Protomagdalénien restent donc inexpliqués.
Les séries gravettiennes et protomagdaléniennes d'Auvergne se caractérisent par l'absence de projectiles en matière animale. Ceci est à mettre très probablement en relation avec l'abondance générale des armatures lithiques qui pourraient avoir pris la place des traits en bois de renne, avec des hampes en bois végétal. Ce phénomène, qui pourrait s'expliquer sur le gisement du Sire par la très forte domination du Cheval dans la faune chassée, concerne également, et de façon plus surprenante, le Blot, où le Renne est pourtant l'animal dominant.

En dernier lieu, il faut souligner l'importance qu'occupent les matières premières importées à longue distance dans l'industrie lithique des chasseurs-cueilleurs du Gravettien et surtout du Protomagdalénien d'Auvergne. Ce trait nous semble a priori explicable d'abord par des raisons d'ordre social et non technique. Comme nous l'avons déjà montré par ailleurs, il caractérise l'ensemble des industries lithiques du Paléolithique supérieur et du Néolithique d'Auvergne.

Addendum : II nous est agréable de rendre hommage à la mémoire d'Henri Delporte, récemment disparu, qui a été l'initiateur de la fouille du gisement du Blot. La publication de cette recherche est coordonnée par J.-P. Daugas.

\section{BIBLIOGRAPHIE}

AUBRY T. - 1991 - L'exploitation des ressources en matières premières lithiques dans les gisements solutréens et badegouliens du bassin versant de la Creuse (France). Thèse de Doctorat en Préhistoire, Université de Bordeaux I.

BERTRAN P. - Sous presse - Dépôts de pente continentaux, dynamiques et faciès. Quaternaire, hors-série $n^{\circ} 1$.

BORDES F. 1968 - La question Périgordienne. In : La Préhistoire, problèmes et tendances, éd. du CNRS, p. 59-70.

BORDES F. 1978 - Le protomagdalénien de LaugerieHaute-Est (fouilles F. Bordes). Bull. Soc. Préh. Fr., t. 75, 1112 , p. $501-521$.

BORDES F. et SONNEVILLE-BORDES D. (de). 1966 Protomagdalénien ou Périgordien VII ? L'Anthropologie, t. $70, \mathrm{n}^{\circ} 1-2$, p. 113-122.

BORDES J.-G. et LENOBLE A. 2002 - La « lamelle Caminade ": un nouvel outil lithique aurignacien ? Bull. Soc. Préh. Fr., t. 99, n 4, p. 735-749.

BOSSELIN B. 1992 - Les industries lithiques du protomagdalénien à partir des données du site du Blot (Cerzat, Haute-Loire). Thèse de doctorat de l'université de Franche-Comté, 3 t., 473 p., 108 fig., 57 tab., dact.

BOSSELIN B. 1997 - Le protomagdalénien du Blot. ERAUL 64, Liège. $321 \mathrm{p}$. 
BRACCO J.-P. 1992 - Le paléolithique supérieur du Velay et de ses abords. Recherches sur la dynamique de peuplement et l'occupation du sol dans un milieu volcanique de moyenne montagne. Thèse de doctorat de l'université d'Aix-Marseille, 229 p., dact.

BRACCO J.-P. 1996 - Du site au territoire. L'occupation du sol dans les hautes vallées de la Loire et de l'Allier au paléolithique supérieur (Massif central). Gallia Préhistoire, 38 , p. $43-67$.

BRICKER H.-M. 1995 - Le paléolithique supérieur de l'abri Pataud (Dordogne), les fouilles de H.L. Movius. Documents d'Archéologie Française, Paris, éditions de la Maison des Sciences de l'Homme, 328 p., 82 fig.

BUISSON D. 1991 - Le Périgordien du Blot (Cerzat, Haute-Loire). Note préliminaire. Bull. Soc. Préh. Fr., t. 88, $\mathrm{n}^{\circ} 4$, p. 104-108.

CHAUVIĖRE F.-X. et FONTANA L. 2005 - Modalité d'exploitation des rennes du Blot (Haute-Loire) : entre subsistance technique et symbolique. In Dujardin V. (dir.) - Industrie osseuse et parures du Solutréen et Magdalénien en Europe. Mémoire $\mathrm{n}^{\circ}$ 39 de la Société Préhistorique Française, pp. 137-148.

CLAY R.- B. 1995 - Le Protomagdalénien de l'abri Pataud, niveau 2. In : BRICKER (dir.) Le Paléolithique supérieur de l'abri Pataud (Dordogne), les fouilles de H.L. Movius. Documents d'Archéologie Française, Paris, éditions de la Maison des Sciences de l'Homme, p. 67-87.

COSTAMAGNO S., THÉRY-PARISOT I., BRUGAL J.-P. et GUIBERT R. 2005 - Taphonomic consequences of the use of bones as fuel. Experimental data and archaeological applications. In : O'Connor T. (dir.) - Biosphere to Lithopshere. New studies in vertebrate taphonomy, Actes du $9^{\circ}$ Colloque de l'ICAZ, Durham, août 2002, Oxford, Oxbow Books, p. 51-62.

DEBOUT G. 2003 - Les microlithes du Magdalénien supérieur du Bassin Parisien : une diversité inattendue. In : Ladier E. - Les pointes à cran dans les industries lithiques du Paléolithique supérieur récent, Préhistoire du SudOuest, suppl. $\mathrm{n}^{\circ}$ 6, p. 91-99.

DELPORTE H. 1972 - Protomagdalénien du Blot, étude préliminaire. Congrès Préhistorique de France, $\mathrm{XIX}^{\ominus}$ session, pp. 190-199.

DELPORTE H. 1976 - Les civilisations du Paléolithique supérieur en Auvergne. La Préhistoire française, t. 1, vol. 2, Paris, éditions du C.N.R.S., p. 1297-1304.

DELPORTE H., SURMELY F. et URGAL A. 1999 Châtelperron, un grand gisement préhistorique de l'Allier. Clermont-Ferrand, service régional de l'archéologie d'Auvergne, $49 \mathrm{p}$.

DEMARS P.-Y. 1994 - L'économie du silex au Paléolithique supérieur dans le nord de l'Aquitaine. Thèse de doctorat d'Etat de l'université de Bordeaux 1, 2 t., 549 et 270 p., dact.
D'ERRICO F., VANHAEREN M., SANCHEZ GONI M., GROUSSET F., VALLADAS H. et RIGAUD J.-Ph. 2001 Les possibles relations entre l'art des cavernes et la variabilité climatique rapide de la dernière période glaciaire. In : Barrandon J.-N., GUIBERT P. et MICHEL V. (dir.) : Datation. XXlèmes Rencontres internationales d'archéologie et d'histoire d'Antibes, ed. APDCA, p. 333-347.

DIGAN M. 2001 - Le gisement gravettien de la Vigne-Brun (Loire) : première étude de l'industrie lithique de l'unité $K L 19$. Thèse de doctorat de l'université de Lille 1, 2 vol., $465 \mathrm{p}$.

DJINJIAN F., KOSLOWSKI J. et OTTE M. 1999 - Le paléolithique supérieur en Europe. Armand Colin, Paris, 474 p.

DUFRESNE N. 1999 - La discrimination des silex tertiaires auvergnats : étude géologique et application archéologique (magdalénien). Mémoire de DESS de l'université de Dijon, 87 p., dact.

FONTUGNE M. 2004 - Les derniers progrès du calibrage des âges radiocarbones permettent-ils une révision des chronologies entre 25.000 et 50.000 BP ? Quaternaire, vol. $15, n^{\circ} 3$, p. $245-252$.

FOUCHER J.-C. 1979 - Distribution stratigraphique des kystes de dinoflagellés et des acritarches dans le Crétacé supérieur du bassin de Paris et de l'Europe septentrionale. Palaeontographica, 169, fasc. 1-3, p. 78-105.

GERVAIS Ph. 2001 - Les silex géologiques du turonien de Touraine, caractérisation pétrologique et géochimique. Comparaison avec des artefacts auvergnats. Mémoire de maîtrise de l'université Blaise Pascal de ClermontFerrand, $28 \mathrm{p}$.

GIEZ S. 2001 - Recherche sur l'origine des silex archéologiques d'Auvergne : comparaison avec des silex du Bassin de Paris. Mémoire de maîtrise de l'université Blaise Pascal de Clermont, $25 \mathrm{p}$.

GIOT D., MALLET N. et MILLET D. 1986 - Les silex de la région du Grand-Pressigny. Recherche géologique et analyse pétrographique. Revue archéologique du Centre de la France, t. 25, vol. 1, p. 21-36.

HAHN J. 1993 - Geschichtliche Forschung der Ostalb. Karst und Höhle, p. 213-224.

HAYS M. et SURMELY F. 2005 - Réflexions sur la fonction des microgravettes et la question de l'utilisation de l'arc au Gravettien ancien. Paléo, n 17, p. 145-155.

HEINRICH H. 1988 - Origin and consequences of cyclic ice rafting in the northeast Atlantic Ocean during the past 130.000 years. Quaternary Research, 29, p. 142-152.

IRRIBARIA R. 1992 - La diffusion de l'opalite dans les pays de la Loire moyenne. Etat de la question en 1992. Mémoire de DEA, université de Paris 1, 60 p., dact. 
KLARIC L. 1999 - Un schéma de production lamellaire original dans l'industrie gravettienne de l'ensemble moyen du gisement du Blot à Cerzat (Haute-Loire). Mémoire de DEA de l'université de Paris 1, 2 vol, dact.

KLARIC L. 2000 - Note sur la présence de lames aménagées par technique de Kostienki dans les couches gravettiennes du Blot (Cerzat, Haute-Loire). Bull. Soc. Préh. Fr., t. $97, n^{\circ} 4$, p. $625-636$.

KLARIC L. 2003 - L'unité technique des industries à burins du Raysse dans leur contexte diachronique. Réflexions sur la diversité culturelle au Gravettien à partir des données de la Picardie, d'Arcy-sur-Cure, de Brassempouy et du Cirque de la Patrie. Thèse de doctorat de l'université de Paris 1, $426 \mathrm{p}$.

KLARIC L., AUBRY T., WALTER B. 2002 - Les lamelles de la Picardie, un nouveau type d'armature en contexte gravettien et son mode de production: les « burins du Raysse " (La Picardie, commune de Preuilly-Sur-Claise, Indre-et-Loire). Bulletin de la Société préhistorique Française, t. 99, n 4, p. 751-764.

KONG-CHO S.-J. - 1997 - Etude de l'industrie lithique de la couche 2 dite protomagdalénienne de l'abri Pataud, Les Eyzies, Dordogne : étude technologique et typologique. Thèse de doctorat du Muséum national d'Histoire naturelle, Institut de Paléontologie humaine, 374 p., ill.

KOZLOWSKI J. et LENOIR M. 1988 - Analyse des pointes à dos des gisements périgordiens de l'Aquitaine. Universitas lagellonica, Acta Scientarum Literrarumque, $n^{\circ}$ 43, 94 p.

LACORRE F. 1960 - La Gravette, le Gravettien et le Bayacien. Laval, ed. Barnéoud, 369 p, 26 fig.

LÉOZ L. 2001 - Contribution à l'étude du gravettien de l'abri Pataud (Dordogne) : étude techno-typologique de l'industrie lithique du niveau 5 : Front, attribuée au périgordien IV. Bulletin de la Société Historique et Archéologique du Périgord, t. 128, p. 581-596.

MARGUERIE D. 1982 - Étude géologique du gisement préhistorique du Blot (Cerzat, Haute-Loire) : éléments de lithostratigraphie et paléoécologie. D.E.S. de l'EHESS, 261 p, 78 fig., 6 pl.

MASSON A. 1981 - Pétroarchéologie des roches siliceuses, intérêt en préhistoire. Thèse de 3ème cycle. Univ. de Lyon I, $101 \mathrm{p}$.

MASSON A. 1983 - Les territoires et la circulation des silex au paléolithique supérieur. Bulletin de la Société Lynéenne de Lyon, 52ème année, $n^{\circ} 7$, p. 207-217.

MASSON A. 1986 - Nouvelles contributions aux études pressigniennes. Revue Archéologique de l'Ouest, sup. 1, p. 111-120.

MAUGER M. 1985 - Les matériaux siliceux utilisés au paléolithique supérieur en lle de France : occupation du territoire, déplacements et approche des mouvements saisonniers. Thèse de 3ème cycle, université de Paris 1, 294 p.

MELLARS P. 2004 - Neanderthals and the modern human colonization of Europe. Nature, vol. 432, p. 431-465.

MORALA A. 1999 - Grandes pièces arquées du magdalénien supérieur : une nouvelle préforme de grand nucléus à lames découverte en Lot-et-Garonne. Paléo, $\mathrm{n}^{\circ} 11$, p. 199-209.

NESPOULET R., CHIOTTI L., HENRY-GAMBIER D., AGSOUS S., LENOBLE A., MORALA A., GUILLERMIN P. et VERCOUTÈRE C. 2008 - L'occupation humaine de l'abri Pataud (Les Eyzies-de-Tayac, Dordogne) il y a 22000 ans : problématique et résultats préliminaires des fouilles du niveau 2. In : JAUBERT J., BORDES J.-G., ORTEGA I. Les sociétés du Paléolithique dans un Grand Sud-Ouest de la France : nouveaux gisements, nouveaux résultats, nouvelles méthodes. Mémoire de la Société Préhistorique Française, XLVII, Paris, p. 325-334.

OTTE M. 1980 - Le gravettien en Europe centrale. Dissertationes Archaeologicae Gandenses, 20, 2 vol.

PASTY J.-F., BALLUT Ch., MURAT R. et ALIX Ph. Soumis Le gisement moustérien des Chausses à Mirefleurs (Puyde-Dôme, France). Cent ans de Préhistoire et de Protohistoire dans le Massif central, bilans et perspective. Actes du colloque du Puy-en-Velay, novembre 2004.

PELEGRIN J. 1992 - Quelques remarques sur le débitage. In : Schmider B. : Marsangy, un campement des derniers chasseurs magdaléniens sur les bords de l'Yonne. ERAUL 55 , Liège, p. 108-115.

PEYRONY D. et E. 1938 - Laugerie-Haute. Archives de I'IPH, mémoire 19, $81 \mathrm{p}$.

PIGEOT N. 2004 (dir.) - Les derniers magdaléniens d'Étiolles. Perspectives culturelles et paléohistoriques. XXXVII ème supplément à Gallia Préhistoire, $351 \mathrm{p}$.

SONNEVILLE-BORDES D. (de) 1960 - Le Paléolithique supérieur en Périgord. Bordeaux, Delmas, 2 vol., 560 p.

SORIANO S. 1998 - Les microgravettes du périgordien de Rabier à Lanquais (Dordogne). Analyse technologique fonctionnelle. Gallia Préhistoire, t. 40, p. 75-94.

SURMELY F. 1998 - Le peuplement de la moyenne montagne auvergnate, des origines à la fin du mésolithique. Thèse de doctorat de l'université de Bordeaux 1. 2 t., 239 et $205 \mathrm{p}$, dact.

SURMELY F. 2000 - Le peuplement magdalénien de l'Auvergne. Essai de synthèse. In : G. Pion (Ed.), Le paléolithique supérieur récent : nouvelles données sur le peuplement et le paléoenvironnement, actes de la table ronde de Chambéry, 1998. Mémoire de la Société Préhistorique Française, $\mathrm{n}^{\circ} 17$, p. 165-176. 
SURMELY F. 2004 - Les sources minérales oubliées du Massif-Central. Ollierguex, éd. de la Montmarie.

SURMELY F. 2009, ce vol. - La diffusion du silex crétacé dans le centre du Massif Central (Auvergne, Velay, forez) durant la Préhistoire. Colloque du CTHS, La Rochelle, avril 2004.

SURMELY F. et ALIX Ph. 2005 - Note sur les talons en éperon du Protomagdalénien. Paléo, n 17, p. 157-176.

SURMELY F., ALIX Ph., COSTAMAGNO S., DANIEL P., MURAT R., RENARD R. et VIRMONT J. 2003 - Découverte d'un gisement du gravettien ancien au lieu-dit Le Sire, commune de Mirefleurs, Puy-de-Dôme. Bull. de la Soc. Préhist. Fr., t. 100, p. 29-40.

SURMELY F. et MURAT R. 2003 - Études comparatives sur la résistance mécanique de divers silex lacustres et marins français. Actes de la table ronde internationale d'Aurillac "les matières premières lithiques en préhistoire". Supplément à Préhistoire du Sud-Ouest, p. 337-342.

SURMELY F., BRACCO J.-P., BARRIER P., CHARLY N. et LIABEUF R. 1998 - Caractérisation des matières premières siliceuses par l'étude des microfaciès et application à la connaissance du peuplement préhistorique de l'Auvergne. ComptesRendus de l'Académie des Sciences de Paris, 326, p. 595-601.

SURMELY F., PASTY J.-F., ALIX Ph., MURAT R. et LIABEUF R. 2002 - Le gisement magdalénien du Pont-deLongues (Les-Martres-de-Veyre, Puy-de-Dôme). B. S. P. F., t. 99, pp. 13-38.

SURMELY F. et PASTY J.-F. 2003 - Les importations de silex en Auvergne durant la préhistoire. Actes de la Table ronde internationale d'Aurillac "les matières premières lithiques en préhistoire". Supplément à Préhistoire du SudOuest, p. 327-336.
SURMELY F., VIRMONT J. et QUINQUETON A. - sous presse - Le gisement épipaléolithique ancien de la grotte Béraud (Haute-Loire). Actes de la table ronde internationale sur l'épipaléolithique et le mésolithique de Tours, 2001.

THÉRY-PARISOT I. et COSTAMAGNO S. sous presse Propriétés combustibles des ossements : données expérimentales et réflexions archéologiques sur leur emploi dans les sites paléolithiques. Gallia Préhistoire.

VALENSI L. 1957 - Micropaléontologie des silex du GrandPressigny. Bulletin de la Société Géologique de France, 6ème série, t. 7, p. 1083-1090.

VALENTIN B. 1995 - Les groupes humains au Tardiglaciaire dans le Bassin Parisien. Thèse de doctorat de l'université de Paris 1, 3 t., dact.

VANHAEREN M. et d'ERRICO F. 2003 - Le mobilier funéraire de la Dame de Saint-Germain-la-Rivière et l'origine paléolithique des inégalités. Paléo, $\mathrm{n}^{\circ} 15, \mathrm{p}$. 195-238.

VIRMONT J. 1981 - Le bassin de l'Allier au paléolithique supérieur. industries et phases de peuplement. Thèse de 3ème cycle de l'université d'Aix-Marseille., 378 p.

ZILHAO J. et D’ERRICO F. 2003 - An Aurignacian « Garden of Eden » in southern Germany ? An alternative interpretation of the Geissenklösterle and a critique of the Kulturpumpe model. Paléo, $\mathrm{n}^{\circ} 15$, p. 69-86. 